IUHET 134

CCNY-HEP-87/6

June, 1987

\title{
COVARIANT STRING AMPLITUDES \\ ON EXOTIC TOPOLOGIES \\ TO ONE LOOP
}

V. Alan Kostelecký, ${ }^{a}$ Olaf Lechtenfeld, ${ }^{b}$ and Stuart Samuel ${ }^{c}$

\author{
${ }^{a}$ Physics Department \\ Indiana University \\ Bloomington, IN 47405, U.S.A. \\ ${ }^{b}$ Theoretical Division \\ CERN \\ 1211 Genève 23 \\ Switzerland \\ ${ }^{c}$ City College of New York \\ 138th and Convent Ave. \\ New York, NY 10031, U.S.A.
}

\begin{abstract}
Rules are provided for the calculation of string amplitudes on all exotic topologies to one loop. These topologies include the real projective plane, the disk, the annulus, the Möbius strip, the torus and the Klein bottle. The rules are valid for bosonic strings, superstrings, and heterotic strings either in their critical dimensions or toroidally compactified, and they are applicable to amplitudes with arbitrary external bosonic and fermionic states, including mixed open- and closed-string transitions. The modular invariance of the torus amplitudes is discussed.
\end{abstract}




\section{INTRODUCTION}

\section{A. General Background}

The anomaly cancellation [1] in certain superstring theories $[1,2]$ and the possibility that these same theories are finite [1-6] has generated an enormous interest in loop corrections to string amplitudes. Early computations of one-loop effects can be found in refs. [7-26] and are summarized in ref. [27]. With the advent of the Polyakov approach [28, 29], further progress has been made [30-63].

The development of conformal field theory [64-75] has also led to improvements at treelevel, especially for fermionic processes. A set of rules for obtaining correlation functions for tree-level amplitudes in open or closed, bosonic or supersymmetric strings and in heterotic strings in their critical dimensions has been achieved [76-78].

In the modern approach, BRST invariance plays a crucial role $[79,80]$. In quantizing the string, gauge symmetries associated with reparametrization invariances must be fixed. This leads to the ghost fields $b(\sigma, \tau)$ and $c(\sigma, \tau)$. In the supersymmetric case, superconformal invariance leads to additional ghosts, $\beta(\sigma, \tau)$ and $\gamma(\sigma, \tau)[73]$. These bosons can be "bosonized" in terms of fermions and a bosonic field $\phi$ [73]. The latter naturally combines with the Neveu-Schwarz-Ramond (NSR) field, $\psi^{\mu}$, to form the ghost-extended superstring as discussed in [78] and elaborated in refs. $[81,82]$.

The purpose of the present paper is to extend the formalism developed in [73] beyond the tree-level. We treat closed-string amplitudes on the real projective plane and on the disk, and mixed open- and closed-string transitions on the disk. For the open string, oneloop effects involve the annulus and Möbius strip, while for the closed string, the torus and Klein bottle also appear. Some of these amplitudes have been discussed from the light-cone viewpoint in [27].

Propagators on these various topologies are obtained and correlation functions are determined. For each of the above-mentioned topologies, a set of rules is provided from 
which one can compute any amplitude.

Explicit calculations are greatly simplified by bosonization of certain fields [73]. The bosonization procedure we use is given in ref. [78]. The key formula is

$$
O^{\lambda}(z)=\exp (\lambda \cdot \phi) c_{\lambda}
$$

where $c_{\lambda}=\exp \left(i \pi \lambda \cdot M[\partial \phi]_{0}\right)$ is a cocycle operator. The $N \times N$ matrix $\mathrm{M}$, whose entries are 0 and \pm 1 , is given in ref. [78]; $\left[\partial \phi^{i}\right]_{0}$ is the zero mode of $\partial \phi^{i}$, and $\lambda \cdot \phi=\lambda_{i} \phi^{i}$. Eq. (I.1) covers a variety of situations depending on the number of bosons and the nature of the $\lambda$ vectors. For example, the $\mathrm{b}$ and $\mathrm{c}$ reparametrization ghosts require only one boson, so $\mathrm{N}=1$ [73]. For the ghost-extended superstring system, $N=6$. The first five bosons describe the NSR field $\psi^{\mu}$, while $\phi^{6}$ is the superconformal ghost $\phi$ mentioned above. When $\lambda_{i}= \pm \frac{1}{2}$ for all $i$, covariant spin fields are obtained. For an $o(2 \mathrm{~N})$ algebra, there are $\mathrm{N}$ bosons and Eq. (I.1) can be used to represent the spin fields for the internal groups associated with heterotic strings. When $\lambda_{i}$ are different from $\pm \frac{1}{2}$, the bosonization of other fields is achieved. For example, when $\lambda$ is $\pm e_{k} \pm e_{\ell}$, where $e_{k}$ is a unit vector in the kth direction, Eq. (I.1) becomes the vertex-operator construction [83-91].

Bosonization for higher-genus systems has been discussed in refs. $[36,41,50,51,54$, $73,92-94]$. There have been suggestions that the bosonization procedure depends on the type of surface. We find that to the one-loop level Eq. (I.1) is sufficient. In fact, one must even use the same normal-ordering procedure used at tree level. The same applies to exponentials of the $X^{\mu}$ fields. Exponentials of bosons, if not normal-ordered, give rise to infinite self-energy contributions. The safest procedure is to introduce a cut-off and multiplicatively renormalize the operators. At tree level, this is equivalent to normal ordering.

\section{B. Exotic Topologies}

In general, an arbitrary two-dimensional surface is topologically equivalent to a sphere with $\mathrm{B}$ holes, $\mathrm{C}$ cross-caps, and $\mathrm{H}$ handles [95]. The Euler characteristic $\chi$ is then given 
by $\chi=2-B-C-2 H$ [96]. In this paper, we are concerned with string amplitudes for external-state emission from surfaces with topologies of Euler characteristic $\chi=1$ or 0 . For $\chi=1$, these include the real projective plane $R P^{2}$ and the disk; for $\chi=0$, they include the annulus, the Möbius strip, the torus and the Klein bottle. The annulus is sometimes called the cylinder or the ribbon. Of these surfaces, the real projective plane, the Möbius strip and the Klein bottle are nonorientable. In practice, this means that the orientation of a spinor or a vector can be reversed by parallel transport around a noncontractible loop. Such surfaces contribute only to amplitudes in unoriented string theories [27].

From unitarity arguments, the order in the string coupling constant $\mathrm{g}$ to which a given amplitude contributes is determined by the topology of the associated surface and by the number and type of external states emitted $[97,45]$. For a surface of Euler characteristic $\chi$ with $\mathrm{m}$ open-string states and $\mathrm{n}$ closed-string states emitted, the amplitude contributes at order $g^{m+2 n-2 \chi}$. $^{*}$

For nonorientable surfaces, one might expect an obstruction to chiral bosonization [98]. For example, parallel transport of a right-moving boson around a noncontractible loop in the Möbius strip results in a left-moving boson. In principle, this could be an issue for the bosonization of fermions such as the b-c ghosts, the spin fields, or the NSR fields. In practice, we find no difficulty. For example, the Möbius strip may be viewed as a flat strip that is twisted before its ends are joined. The joining process can be represented by a vertex function. In the presence of this vertex function, a left-moving field can be replaced by a right-moving one; furthermore, at the junction it can be replaced precisely by its right-moving counterpart.

* This means the terminology "tree" or "one loop" must be applied with caution. For example, the disk is a tree amplitude from the viewpoint of open-string emission, but it is intermediate between tree and one loop, i.e., it is a higher-order tree, from the viewpoint of closed strings. 
A variety of methods have been employed to determine correlation functions for the higher-order amplitudes, as follows.

(1) The method of images. A correlation function or propagator is related to a sum of known correlation functions or propagators for which the $\tau$ and $\sigma$ coordinates are displaced. (2) Using a vertex function or boundary operator. These are functionals of the oscillators implementing the appropriate topology.

(3) Explicit evaluation of the trace at the one-loop level. The functional integral is determined by relating it to $\operatorname{tr}\left(e^{-\tau H}\right)$, which is then evaluated by summing over a complete set of states.

(4) Direct evaluation of the functional integral. The functional integral must be calculated over the particular topology in the presence of vertex operators. A field is decomposed into a classical piece and a quantum piece. The classical piece satisfies the free equation of motion with boundary conditions. This equation must be solved. The quantum piece is treated with standard functional-integral methods.

(5) Solving the equation for the propagator. Many correlation functions can be obtained via Wick's theorem if the propagators are known.

(6) Using complex function theory. The behavior of a correlation function under transformations of its complex arguments $z_{i}$ may suffice to determine it if the poles and zeros are known. This is particularly useful if the correlation function has periodicity properties in its arguments, as for the torus case.

(7) The conformal-mapping method. Using a conformal map, a given topology is mapped onto a standard one such as the half-plane. Since correlation functions are known for the latter, conformal invariance determines them for the former.

Method (1) is used throughout this paper. Further examples may be found in refs. [45-47]. Sections II and III also make use of method (2); examples are given in [99- 
101]. Sections IV and V use methods (3)-(5). Method (3) is frequently used in light-cone calculations [27]. It is useful to perform an oscillator decomposition before applying (3)(5), as a system then separates into a series of decoupled harmonic oscillators. It is also a good idea to separate zero modes from oscillators. Method (4) has been applied in [51, 52]. Method (6) has recently been exploited for higher-genus closed-string topologies [58-59]. Method (7) is also a potentially useful method for arbitrary genus. The latter two are not discussed in this paper because methods (1)-(5) suffice. Although our primary goal is to present the rules and results for correlation functions, in selected places we have indicated the techniques applied.

The implementation of projections by summation over certain topologies is an important and recurring theme. Thus, two tori with different twist angles, $\theta$, represent different topologies; integrating over $\theta$ enforces the closed-string constraint $L_{0}^{L}-L_{0}^{R}=0$. Alternatively, a sum over spin structures implements the GSO projection [103]. Another example is the projection to unoriented closed strings from oriented ones. This can be represented by a projection operator, $(\mathrm{I}+\mathrm{T}) / 2$, where $\mathrm{I}$ is the identity operator and $\mathrm{T}$ is a certain left-right interchange operator [27]. At the one-loop level, the I term corresponds to the torus and the $\mathrm{T}$ term to the Klein bottle; their sum imposes a projection onto the restricted closed-string spectrum. A similar phenomenon takes place for open strings with the torus and Klein bottle replaced by the annulus and Möbius strip. One consequence is that a treatment of the individual surfaces in the Polyakov sum may not be the most appropriate organization of the loop expansion. Certain terms need to be grouped. Finiteness of the series can depend on cancellations between different terms [1, 3-6, 47, 53, 104-106].

Oscillator expansions for $X_{\text {open }}(\sigma, \tau)$ are 


$$
\begin{aligned}
X_{o p e n}^{\mu}(\sigma, \tau) & =i x^{\mu}+2 \alpha^{\prime} p^{\mu} \tau+\sqrt{2 \alpha^{\prime}} \sum_{n \neq 0} \frac{\alpha_{-n}^{\mu} \cos n \sigma e^{n \tau}}{n} \\
& \equiv \frac{1}{2}\left[X^{\mu}(z)+X^{\mu}(\bar{z})\right], \\
X^{\mu}(z) & =i x^{\mu}+2 \alpha^{\prime} p^{\mu} \ln z+\sqrt{2 \alpha^{\prime}} \sum_{n \neq 0} \frac{\alpha_{-n}^{\mu} z^{n}}{n} .
\end{aligned}
$$

Furthermore,

$$
\begin{aligned}
\partial X^{\mu}(z) & =\sqrt{2 \alpha^{\prime}} \sum_{n=-\infty}^{\infty} \alpha_{-n}^{\mu} z^{n-1} \\
& =2 \partial X_{o p e n}^{\mu}(\sigma, \tau) \\
& =\frac{1}{2 z}\left(\frac{\partial}{\partial \tau}-i \frac{\partial}{\partial \sigma}\right)\left(2 X^{\mu}(\sigma, \tau)\right)
\end{aligned}
$$

For closed strings, we have

$$
\begin{aligned}
X_{\text {closed }}^{\mu}(\sigma, \tau) & =i x^{\mu}+\alpha^{\prime} p^{\mu} \tau+\sqrt{\frac{\alpha^{\prime}}{2}} \sum_{n \neq 0} \frac{1}{n}\left(\alpha_{-n}^{L \mu} z^{n}+\alpha_{-n}^{R \mu} \bar{z}^{n}\right) \\
& =X^{L \mu}(z)+X^{R \mu}(\bar{z}), \\
X^{L \mu}(z) & =\frac{i x^{\mu}}{2}+\frac{\alpha^{\prime}}{2} p^{\mu} \ln z+\sqrt{\frac{\alpha^{\prime}}{2}} \sum_{n \neq 0} \frac{1}{n} \alpha_{-n}^{L \mu} z^{n} \\
X^{R \mu}(\bar{z}) & =\frac{i x^{\mu}}{2}+\frac{\alpha^{\prime}}{2} p^{\mu} \ln \bar{z}+\sqrt{\frac{\alpha^{\prime}}{2}} \sum_{n \neq 0} \frac{1}{n} \alpha_{-n}^{R \mu} \bar{z}^{n}
\end{aligned}
$$

and

$$
\begin{aligned}
& \partial X^{L}(z)=\sqrt{\frac{\alpha^{\prime}}{2}} \sum_{n} \alpha_{-n}^{L} z^{n-1}, \quad \alpha_{0}^{L}=\sqrt{\frac{\alpha^{\prime}}{2}} p=\alpha_{0}^{R}, \\
& \partial X^{R}(\bar{z})=\sqrt{\frac{\alpha^{\prime}}{2}} \sum_{n} \alpha_{-n}^{R} \bar{z}^{n-1} .
\end{aligned}
$$

To avoid confusion when dealing with both open and closed strings, the slope parameter $\alpha^{\prime}$ is explicitly displayed. Note that $X^{L}(z)$ and $X^{R}(\bar{z})$ have common center-of-mass coordinates and momenta. It is sometimes convenient to replace them by equivalent fields $X_{e q}^{L}(z)$ and $X_{e q}^{R}(\bar{z})$ which have independent zero-mode coordinates: 


$$
\begin{aligned}
& X_{e q}^{L}(z)=i x^{L}+\frac{\alpha^{\prime}}{2} p^{L} \ln z+\sqrt{\frac{\alpha^{\prime}}{2}} \sum_{n \neq 0} \frac{1}{n} \alpha_{-n}^{L} z^{n}, \\
& X_{e q}^{R}(\bar{z})=i x^{R}+\frac{\alpha^{\prime}}{2} p^{R} \ln \bar{z}+\sqrt{\frac{\alpha^{\prime}}{2}} \sum_{n \neq 0} \frac{1}{n} \alpha_{-n}^{R} \bar{z}^{n} .
\end{aligned}
$$

In certain situations, a truly chiral decomposition of $X_{\text {closed }}(\sigma, \tau)$ can be made. This means that $X_{\text {closed }}(\sigma, \tau)$ can be represented by $X_{e q}^{L}(z)+X_{e q}^{R}(z)$ in lieu of Eq. (I.4).

In Eqs. (I.2-1.6), $z=\exp (\tau+i \sigma)$. Other useful variables are

$$
\begin{aligned}
& \rho=\ln z=\tau+i \sigma, \\
& \bar{\rho}=\ln \bar{z}=\tau-i \sigma, \\
& \nu=\frac{\rho}{2 \pi i}, \\
& \bar{\nu}=\frac{-\bar{\rho}}{2 \pi i} .
\end{aligned}
$$

Our notational conventions are given in refs. $[78,107]$. The abbreviations NS for NeveuSchwarz and $R$ for Ramond are used. The subscripts or superscripts $L$ and $R$ refer to left and right. The state $|\Omega\rangle$ denotes the $\mathrm{SL}(2, \mathrm{R})$ - or $\mathrm{SL}(2, \mathrm{C})$-invariant vacuum $[73]$. Throughout this paper we use a Cartan-Weyl basis for the Lorentz group. Therefore, amplitudes are not expressed in terms of manifestly Lorentz-covariant notation. The results, however, are covariant: data from a scattering process in ten dimensions among particles of certain helicities could readily be compared to our results. Using the relation between the two bases given in ref. [78], one simply constructs the appropriate linear combination of amplitudes.

This paper is organized as follows. Section II discusses closed string propagation on $R P^{2}$ and on the disk. Section III treats transitions between open and closed strings. Amplitudes involving the annulus and the Möbius strip are the subject of Section IV, while those involving the torus and Klein bottle are discussed in Section V. For each topology, a set of rules and a series of techniques for calculating correlation functions is provided. Section VI discusses modular invariance, while Section VII contains a summary 
and discussion. The Appendix presents some harmonic-oscillator results applicable with methods (3)-(5) above and derives the non-holomorphic term appearing in the one-loop $X^{\mu}$ propagator. 


\section{II. $R P^{2}$ AND DISK AMPLITUDES: CLOSED-STRING SCATTERING}

\section{A. Introduction}

This Section examines the emission of both bosonic and fermionic closed strings from $R P^{2}$ and from the disk. To implement the calculations, we define spin fields on these two topologies via bosonization.

We were influenced by the works of Callan, Lovelace, Nappi and Yost [99] and Polchinski and Cai [100]. These authors develop boundary operators implementing the appropriate conformal properties for $R P^{2}$ and disk topologies. By treating the theory in bosonized form and by using the ghost-extended lattice viewpoint [78], we can obtain amplitudes for the emission of arbitrary closed-string states including the more difficult fermionic amplitudes. This Section provides a set of rules and a list of correlation functions from which any closed superstring $R P^{2}$ or disk amplitude may be derived. In Section III, we discuss mixed open- and closed-string scatterings.

\section{B. Boundary Operator}

Let $R=\exp (T)$ be the radius of a disk. From Eq. (I.7), the boundary is located at time $\tau=T$. The identification of opposite boundary points yields the real projective plane, $R P^{2}$. The $R P^{2}$ boundary conditions for $X(\sigma, \tau)$ are $X(\sigma+\pi, T)=X(\sigma, T)$ and $d X(\sigma+\pi, T) / d \tau=-d X(\sigma, T) / d \tau[99]$. These are equivalent to the twisted delta function $\delta\left(X^{R}(\sigma, T)-X^{L}(\pi-\sigma, T)\right)$ that enters in the bilinear form, $\int A * B$, of Witten's string field theory [108, 109] except: (a) the overlap takes place at $T=0$; and (b) for the $X^{\mu}$ degrees of freedom the center-of-mass momenta for the two strings coincide. The result for $\tau \neq 0$ is obtained by applying the evolution operator, $\exp \left(-\tau\left(L_{0}^{L}+L_{0}^{R}\right)\right)$. The difference (b) can be eliminated by employing the equivalent operators in Eq. (I.6). Using the vertex representation in refs. $[107,110-112]$ and identifying Fock spaces 1 and 2 with $L$ and $R$, one gets 


$$
\begin{aligned}
& \langle C, T|=_{X}\left\langle C,\left.T\right|_{\psi-\phi}\langle C, T|{ }_{b-c}\langle C, T|{ }_{\xi-\eta}\langle C, T|\right. \\
& x\langle C, T|={ }_{R}\langle 0| \quad{ }_{L}\langle 0| \exp \left[-\sum_{n=1}^{\infty}(-1)^{n} \frac{\alpha_{n}^{L} \cdot \alpha_{n}^{R} e^{-2 n T}}{n}\right] \\
& \rightarrow \int d^{D} k_{L} d^{D} k_{R} \delta^{D}\left(k_{L}+k_{R}\right) \exp \left[-\alpha^{\prime} R e T\left(k_{L}^{2}+k_{R}^{2}\right)\right] \\
& \times_{R}\left\langle k_{R}\right| \quad{ }_{L}\left\langle k_{L}\right| \exp \left[-\sum_{n=1}^{\infty}(-1)^{n} \frac{\alpha_{n}^{L} \cdot \alpha_{n}^{R} e^{-2 n T}}{n}\right] \text {, } \\
& { }_{b-c}\langle C, T|={ }_{R}\langle\Omega| c_{-1}^{R} \quad{ }_{L}\langle\Omega| c_{-1}^{L}\left(c_{0}^{L}+c_{0}^{R}\right) e^{2 T} \times \exp \left[\sum_{n=1}^{\infty}(-1)^{n}\left(b_{n}^{L} c_{n}^{R}+b_{n}^{R} c_{n}^{L}\right) e^{-2 n T}\right] \\
& =\sum_{\lambda^{n}+\lambda^{L}=3}{ }_{R}\left\langle\lambda^{R}\right| \quad{ }_{L}\left\langle\lambda^{L}\right| \exp \left[-\sum_{n=1}^{\infty}(-1)^{n} \frac{\sigma_{n}^{L} \sigma_{n}^{R}}{n} e^{-2 n T}\right] \\
& \times \exp \left[-\frac{T}{2}\left(\lambda^{L}\left(\lambda^{L}-3\right)+\lambda^{R}\left(\lambda^{R}-3\right)\right)\right] \\
& { }_{\psi-\phi}\langle C, T|=\sum_{\lambda^{R}+\lambda^{L}=(0 ;-2)}{ }_{R}\left\langle\lambda^{R}\right| \quad{ }_{L}\left\langle\lambda^{L}\right| \exp \left[-\sum_{n=1}^{\infty}(-1)^{n} \frac{\left\langle\phi_{n}^{L} \cdot \phi_{n}^{R}\right\rangle}{n} e^{-2 n T}\right] \\
& \times \exp \left[i \pi\left(\lambda^{L} M \lambda^{R}\right)\right] \\
& \times \exp \left[-\frac{T}{2}\left(\lambda^{L i} \lambda^{L i}-\lambda^{L 6}\left(\lambda^{L 6}+2\right)+\lambda^{R i} \lambda^{R i}-\lambda^{R 6}\left(\lambda^{R 6}+2\right)\right)\right], \\
& \xi-\eta\left(C, T \mid={ }_{R}\left\langle\left.\Omega\right|_{L}\langle\Omega|\left(\xi_{0}^{L}-\xi_{0}^{R}\right) \exp \left[\sum_{n=1}^{\infty}(-1)^{n}\left(\xi_{n}^{L} \eta_{n}^{R}+\xi_{n}^{R} \eta_{n}^{L}\right) e^{-2 n T}\right]\right.\right. \\
& =\sum_{\lambda^{R}+\lambda^{2}=1} R\left\langle\lambda^{R}\right| \quad{ }_{L}\left\langle\lambda^{L}\right| \exp \left[-\sum_{n=1}^{\infty}(-1)^{n} \frac{\chi_{n}^{L} \chi_{n}^{R}}{n} e^{-2 n T}\right] \\
& \times \exp \left[-\frac{T}{2}\left(\lambda^{L}\left(\lambda^{L}-1\right)+\lambda^{R}\left(\lambda^{R}-1\right)\right)\right],
\end{aligned}
$$

where $\langle C, T|$ denotes the boundary operator for $R P^{2}$ with radius $\exp (T)$. The second representation in (II.2) corresponds to using equivalent operators, Eq. (I.6). This representation is used in the remainder of the Section and in Section III. Also, for simplicity we write $X^{L}(z)=X_{e q}^{L}(z)$ and $X^{R}(\bar{z})=X_{e q}^{R}(\bar{z})$. In Eq. (II.4), $\langle>\rangle$ denotes a Lorentzian 
inner product of signature $(+++++-)$. Equations (II.2-II.3) and the NS-NS component of Eq. (II.4) were given in $[99,100]$. The bosonized representation in Eq. (II.4) treats all four sectors NS-NS, NS-R, R-NS and R-R simultaneously. In this approach, the NS and $\mathbf{R}$ sectors are united into one mathematical object, the ghost-extended superstring. The calculation of matrix elements in bosonized form is possible, as has been demonstrated in $[101,102,107,111]$. The factor $\exp \left(i \pi\left(\lambda^{L} \cdot M \lambda^{R}\right)\right)$ in Eq. (II.4) is necessary for Lorentz invariance [107]. It generates the appropriate Lorentz-tensor structure between left- and right-moving sectors, as is argued below. The matrix $M$ is given in ref. [78].

The operator $\langle C, T|$ satisfies the following overlap conditions [107]:

$$
0=\langle C, T|\left[\bar{w}^{h} O_{h}^{R}(\bar{w})-z^{h} O_{h}^{L}(-z)\right], \quad z=e^{2 T} / \bar{w}
$$

where $O_{h}^{L}(z)$ is a left-moving operator of weight h, such as $\exp (\lambda \cdot \phi), \partial X^{L \mu}, c^{L}(z)$, or $b^{L}(z)$.

Eq. (II.6) makes sense only for closed-string theories in which there is a symmetry between left-moving and right-moving states, as for example in unoriented strings. For the superstring, this means that the GSO projections in the two Ramond sectors are the same. Thus, the $h_{L}=0$ left-moving spinor has the same chirality as the $h_{R}=0$ right-moving spinor. In this and the next Sections, we discuss these left-right symmetric theories. They include the $\mathrm{SO}(\mathrm{N})$ type I superstrings.

In proving Eq. (II.6), the identities

$$
\begin{aligned}
& \exp \left[i \pi \lambda_{A} \cdot M \lambda_{B}\right]=\exp \left[i \pi \lambda_{B} \cdot M \lambda_{A}\right](-1)^{\left\langle\lambda_{B} \cdot \lambda_{A}\right\rangle}(-1)^{\left\langle\lambda_{A} \cdot \lambda_{A}\right\rangle\left\langle\lambda_{B} \cdot \lambda_{B}\right\rangle}, \\
& \left.\left\langle\lambda_{A} \cdot \lambda_{B}\right\rangle\right|_{\lambda_{B}=-\lambda_{A}+(0 ;-2)} \in 2 Z
\end{aligned}
$$

are needed. For the ghost-extended superstring, Eq. (II.8) is the analog of the condition that the lattice be even. Equation (II.7) allows the constraint $\left|z_{n}\right|>\left|z_{n-1}\right|>\ldots>\left|z_{1}\right|$ to 
be removed so that the $z_{i}$ can be integrated without restriction over the disk.

From the BRST invariance of Witten's bilinear form, $\int\left[Q_{B R S T}(A) * B+(-)^{A} A *\right.$ $\left.Q_{B R S T}(B)\right]=0$, which has been proven for Eqs. (II.1-II.5) at $\tau=0$, it follows that $\langle C, T|\left(Q_{B R S T}^{R}+Q_{B R S T}^{L}\right)=0$ since the evolution operator commutes with $Q_{B R S T}=$ $Q_{B R S T}^{R}+Q_{B R S T}^{L}$. The BRST invariance of $\langle C, T|$ is a necessary physical requirement [99, 100].

The boundary operator for the disk, $\langle B, T|$, is obtained from $\langle C, T|$ as $\langle B, T|=\langle C, T$ $i \pi / 2 \mid$ [99]. The effect of replacing $T$ by $T-i \pi / 2$ in Eqs. (II.1-II.5) is to eliminate the $(-1)^{n}$ factors.

$\langle B, T|$ satisfies

$$
0=\left\langle B, T\left|\left[\bar{w}^{h} O_{h}^{R}(\bar{w})-z^{h} \exp (i \pi h) O_{h}^{L}(z)\right]\right|_{z=\frac{.2 T}{s}}\right.
$$

and is BRST invariant: $\langle B, T| Q_{B R S T}=0$.

C. Rules

Here are the rules for computing a closed-string $R P^{2}$ or disk amplitude.

(1) Use standard closed-string vertex operators. If $\mathrm{m}$ closed strings are involved, choose m-2 integral-type vertices and $2 c^{L} c^{R}$-type vertices. For the superstring, vertex operators must be chosen so that the sum of the left and right $\phi$-ghost numbers is $\mathbf{- 2}$.

(2) For $R P^{2}$ use $\langle C, T|\left(b_{0}^{L}+b_{0}^{R}\right)$ as the left state and $|\Omega\rangle$ for the right state. For the disk use $\langle B, T|\left(b_{0}^{L}+b_{0}^{R}\right)$ as the left state. The relevant correlation function is the expectation of the vertex operators between these states.

(3) Evaluate the correlation functions using the formulae given below, Eqs. (II.10, II.11, II.13-II.15, II.18).

(4) The integration region for the $z_{i}$ is $\left|z_{i}\right|<\exp (T)$, where $z_{i}$ denotes the integration variable associated with the $i^{\text {th }}$ integral-type vertex.

(5) The measure and integration region for the parameter $T$ is $\int_{-\infty}^{\infty} d T$. 
Concerning (1), the vertex operators are generically the product of a left-moving vertex operator and a right-moving vertex operator. Formulae for the integral-type and c-type vertex operators for massless superstring states are given in Eq. (IV.1) of [78]. A $c^{L} c^{R_{-}}$ type vertex means that a left c-type vertex operator is combined with a right c-type vertex operator. There is one exception to this rule: the case of single closed-string emission, $m=1$. The computation of the dilaton-tadpole expectation involves this case. If $O(z, \bar{z})$ is a conformal weight $\left(h_{L}, h_{R}\right)=(1,1)$ operator in the bosonic string theory not involving the $\mathrm{b}$ and $\mathrm{c}$ ghosts, then not only is $c^{L} c^{R} O(z, \bar{z})$ BRST-invariant but $c^{L} \partial c^{L} c^{R} \partial c^{R} O(z, \bar{z})$ is also. Hence, for $m=1$, a $c^{L} \partial c^{L} c^{R} \partial c^{R}$-type vertex operator must be used.

Note the $\left(b_{0}^{L}+b_{0}^{R}\right)$ ghost insertion in rule (2) and that the measure over the parameter $T$ in rule (4) is flat. These two results are related to the BRST decoupling of zero-norm states. This is discussed at the end of this Section.

Let us now provide the correlation functions needed in rule (3). We present the results for the disk case, $\langle B, T|$, since they are used again in Section III. The results for $\langle C, T|$ are obtained by replacing $T$ by $T+i \pi / 2$.

D. Correlation Functions for the Disk and $R P^{2}$

Recall that we are using the equivalent operators in Eq. (I.6) so that $X_{\text {closed }}(\sigma, \tau)$ is replaced by $X_{e q}^{L}(z)+X_{e q}^{R}(\bar{z})$. This helps eliminate some of the subtleties of the zero modes. Correlation functions involving $X_{\text {closed }}(\sigma, \tau)$ are evaluated using Wick's theorem:

$$
\begin{array}{ll}
\underbrace{\phi_{L}(z) \phi_{L}(w)}_{L} \rightarrow & \epsilon \ln (z-w), \\
\underbrace{\phi_{L}(z) \phi_{R}(\bar{w})}_{L} \rightarrow & \epsilon \ln \left(1-z \bar{w} e^{-2 T}\right), \\
\underbrace{}_{\underbrace{}_{R}(\bar{z}) \phi_{L}}(w) \rightarrow & \epsilon \ln \left(1-\bar{z} w e^{-2 T}\right), \\
\underbrace{}_{R}(\bar{z}) \phi_{R}(\bar{w}) \rightarrow & \epsilon \ln (\bar{z}-\bar{w}),
\end{array}
$$

where $|z|>|w|, \phi_{L}$ and $\phi_{R}$ represent $X_{L}^{\mu}$ and $X_{R}^{\mu}$, and $\epsilon=\alpha^{\prime} / 2$. For example, 


$$
\begin{aligned}
\langle B, T| \exp \left[k_{n} \cdot X\left(z_{n}, \bar{z}_{n}\right)\right] & \ldots \exp \left[k_{i} \cdot X\left(z_{1}, \bar{z}_{1}\right)\right]|\Omega\rangle \\
& =\prod_{j>i}\left[\left(z_{j}-z_{i}\right)\left(\bar{z}_{j}-\bar{z}_{i}\right)\right]^{\frac{a^{\prime}}{2} k_{j} \cdot k_{i}} \\
& \times \prod_{j, i}\left(1-z_{j} \bar{z}_{i} e^{-2 T}\right)^{\frac{a^{\prime}}{2} k_{j} \cdot k_{i}} \times \delta^{D}\left(\sum_{j} k_{j}\right) .
\end{aligned}
$$

The Introduction discussed many methods for computing correlation functions. Eq. (II.11) can be computed by the method of images by introducing mirror images at the points $\exp (2 T) / \bar{z}$. Thus, Eq. (II.11) is

$$
\begin{gathered}
\langle\Omega|\left(\frac{e^{T}}{\bar{z}_{1}}\right)^{\frac{1}{2} \alpha^{\prime} k_{1}^{2}} \exp \left[k_{1} \cdot X^{L}\left(\frac{e^{2 T}}{\bar{z}_{1}}\right)\right] \ldots\left(\frac{e^{T}}{\bar{z}_{n}}\right)^{\frac{1}{2} \alpha^{\prime} k_{n}^{2}} \exp \left[k_{n} \cdot X^{L}\left(\frac{e^{2 T}}{\bar{z}_{n}}\right)\right] \\
\times \exp \left[k_{n} \cdot X^{L}\left(z_{n}\right)\right] \ldots \exp \left[k_{1} \cdot X^{L}\left(z_{1}\right)\right]|\Omega\rangle
\end{gathered}
$$

where conservation of momentum, $\sum_{i} k_{i}=0$, is used. Eq. (II.12) follows from Eq. (II.2) and Eq. (II.9) by moving the factor $\exp \left(k \cdot X^{R}(\bar{z})\right)$ to the left and converting it to $\exp \left(k \cdot X^{L}\left(e^{2 T} / \bar{z}\right)\right)$ using $\langle B, T|$. The method of images for $R P^{2}$ yields $T \rightarrow T+i \pi / 2$ in Eq. (II.12).

Derivatives of $X$ can be eliminated from correlation functions as discussed for example in Section IV of [78]. Draw arrows from each $\partial X^{\mu}$ operator to any other operator. For each Wick contraction associate the following factors: 


$$
\begin{aligned}
& \partial \underline{\phi}^{L}(z) \exp \left[\lambda \cdot \phi^{L}(w)\right] \rightarrow \frac{\epsilon \lambda}{(z-w)}, \\
& \partial \underbrace{\phi^{L}(z) \exp \left[\lambda \cdot \phi^{R}(\bar{w})\right]} \rightarrow \frac{-\epsilon \lambda \bar{w}}{e^{2 T}-z \bar{w}}, \\
& \bar{\partial} \underbrace{R}(\bar{z}) \exp \left[\lambda \cdot \phi^{R}(\bar{w})\right] \rightarrow \frac{\epsilon \lambda}{(\bar{z}-\bar{w})}, \\
& \bar{\partial} \phi^{R}(\bar{z}) \exp \left[\lambda \cdot \phi^{L}(w)\right] \rightarrow \frac{-\epsilon \lambda w}{e^{2 T}-\bar{z} w}, \\
& \partial \phi^{L}(z) \partial \phi^{L}(w) \rightarrow \frac{\epsilon}{(z-w)^{2}} \\
& \partial \underbrace{L}(z) \bar{\partial} \phi^{R}(\bar{w}) \rightarrow \frac{\epsilon e^{2 T}}{\left(e^{2 T}-z \bar{w}\right)^{2}}, \\
& \bar{\partial} \phi^{R}(\bar{z}) \partial \phi^{L}(w) \rightarrow \frac{\epsilon e^{2 T}}{\left(e^{2 T}-\bar{z} w\right)^{2}}, \\
& \underset{\partial}{\bar{\partial} \phi^{R}(\bar{z}) \bar{\partial} \phi^{R}}(\bar{w}) \rightarrow \frac{\epsilon}{(\bar{z}-\bar{w})^{2}},
\end{aligned}
$$

where, again, $|z|>|w|, \phi_{L}$ and $\phi_{R}$ represent $X_{L}^{\mu}$ and $X_{R}^{\mu}, \epsilon=\alpha^{\prime} / 2$, and $\lambda$ represents $k^{\mu}$. These equations follow by differentiating Eq. (II.10). For a mixed derivative-exponential correlation function, one must sum over all possible ways of taking Wick contractions and then multiply the result by Eq. (II.11). Factors associated with higher derivatives are obtained by differentiating Eq. (II.13).

From rule (1), the relevant $b-c$ correlation function is

$$
\begin{gathered}
{ }_{b c}\left\langle B, T\left|\left(b_{0}^{L}+b_{0}^{R}\right) c^{L}\left(z_{4}\right) c^{R}\left(\bar{z}_{3}\right) c^{L}\left(z_{2}\right) c^{R}\left(\bar{z}_{1}\right)\right| \Omega\right\rangle_{b c} \\
=2\left(z_{4}-z_{2}\right)\left(\bar{z}_{3}-\bar{z}_{1}\right)\left(e^{2 T}-e^{-2 T} z_{4} \bar{z}_{3} z_{2} \bar{z}_{1}\right) \\
{ }_{b c}\left\langle B, T\left|\left(b_{0}^{L}+b_{0}^{R}\right) \partial c^{L} c^{L}(z) \partial c^{R} c^{R}(\bar{w})\right| \Omega\right\rangle_{b c}=2\left(e^{-2 T} z \bar{z} w \bar{w}-e^{2 T}\right) .
\end{gathered}
$$

At tree level, the b-c correlation function provides the correct $\mathrm{SL}(2, \mathrm{C})$-invariant measure. The same is true here. Treating $z$ and $\bar{z}$ as independent variables, the measure and integrand associated with rules (1)-(5) are invariant under 


$$
\begin{aligned}
z_{i} & \rightarrow z_{i}+\left(\epsilon_{4}+i \epsilon_{1}\right) z_{i}-\epsilon_{2} e^{T}+\epsilon_{3} z_{i}^{2} e^{-T}, \\
\bar{z}_{i} & \rightarrow \bar{z}_{i}+\left(\epsilon_{4}-i \epsilon_{1}\right) \bar{z}_{i}-\epsilon_{3} e^{T}+\epsilon_{2} \bar{z}_{i}^{2} e^{-T}, \\
e^{T} & \rightarrow e^{T}\left(1+\epsilon_{4}\right)
\end{aligned}
$$

where $\epsilon_{i}$ are infinitesimal real parameters. The transformations by $\epsilon_{1}, \epsilon_{2}$ and $\epsilon_{3}$ generate the subalgebra of $\mathrm{sl}(2, \mathrm{C})$ that leaves invariant the boundary at $\exp (T)$. The parameter $\epsilon_{4}$ is associated with the scaling transformation of all variables including $\exp (T)$. In calculations of string amplitudes without b-c ghosts, one uses $m$ integral vertices and divides by the volume associated with the symmetries. Fixing $z_{n}, \bar{z}_{n}$ and $z_{1}, \bar{z}_{1}$ and using the FadeevPopov method, one gets a Jacobian factor of

$$
\left|\begin{array}{cccc}
z_{1} & i z_{1} & -e^{T} & z_{1}^{2} e^{-T} \\
\bar{z}_{1} & -i \bar{z}_{1} & \bar{z}_{1}^{2} & -e^{T} \\
z_{2} & i z_{2} & -e^{T} & z_{2}^{2} e^{-T} \\
\bar{z}_{2} & -i \bar{z}_{2} & \bar{z}_{2}^{2} e^{-T} & -e^{T}
\end{array}\right|,
$$

which, to a factor of $i$, is Eq. (II.14). Hence, the b-c correlation function generates the correct measure factor.

For the $\psi-\phi$ correlation functions, we use the bosonized representation of Eq. (I.1). Eq. (II.10) is valid for $\phi=\phi^{i}$ with $\epsilon=1, i=1, \ldots, 5$, and for $\phi=\phi^{6}$ with $\epsilon=-1$. Thus,

$$
\begin{aligned}
\psi-\phi & \left\langle B, T\left|O^{\lambda_{n}^{L}, \lambda_{n}^{R}}\left(z_{n}, \bar{z}_{n}\right) \ldots O^{\lambda_{1}^{L}, \lambda_{i}^{R}}\left(z_{1}, \bar{z}_{1}\right)\right| \Omega\right\rangle_{\psi-\phi} \\
& =\prod_{j>i}\left\{\left(z_{j}-z_{i}\right)^{\left\langle\lambda_{j}^{L} \cdot \lambda_{i}^{L}\right\rangle}\left(\bar{z}_{j}-\bar{z}_{i}\right)^{\left\langle\lambda_{j}^{R} \cdot \lambda_{i}^{R}\right\rangle} \exp \left[i \pi \lambda_{j}^{L} M \lambda_{i}^{L}+i \pi \lambda_{j}^{R} M \lambda_{i}^{R}\right]\right\} \\
& \times \prod_{j, i}\left[\left(1-\bar{z}_{i} z_{j} e^{-2 T}\right)^{\left\langle\lambda_{j}^{L} \cdot \lambda_{i}^{R}\right\rangle} \exp \left[i \pi \lambda_{j}^{L} M \lambda_{i}^{R}\right]\right\} \\
& \times \exp \left[-T\left(h\left(\sum_{i} \lambda_{i}^{L}\right)+h\left(\sum_{i} \lambda_{i}^{R}\right)\right)\right] \times \delta_{\sum_{i}\left(\lambda_{i}^{L}+\lambda_{i}^{R}\right),\left(0_{i}-2\right)},
\end{aligned}
$$

where $O^{\lambda_{L} \lambda_{n}}(z, \bar{z})=\exp \left(\lambda_{L} \cdot \phi_{L}\right)(z) \exp \left(\lambda_{R} \cdot \phi_{R}\right)(\bar{z})$. The function $h(\lambda)$ is the weight associated with $O^{\lambda}$ : 


$$
h(\lambda)=\frac{1}{2} \lambda^{i} \lambda^{i}-\frac{1}{2} \lambda^{6}\left(\lambda^{6}+2\right) .
$$

The M-matrix [78] dependence in Eq. (II.18) comes from three sources: (a) when left cocycle factors act on exponentials of $\phi_{L}$ fields; (b) when right cocycle factors act on exponentials of $\phi_{R}$ fields; and (c) the explicit M dependence in Eq. (II.4). Note that the correlation function in (II.18) could be computed by the method of images and that the analog of Eq. (II.12) would appear with physical momenta replaced by $\lambda$ vectors. Since a right operator at $\bar{z}$ gets replaced by a left operator at the mirror point $\exp (2 T) / \bar{z}$, one might expect the right cocycle operator to be replaced by the left cocycle operator. Indeed, the explicit $M$ dependence in Eq. (II.4) leads to this result. These M-matrix phases generate the Lorentz-tensor structure in an amplitude [78], so that, for $R P^{2}$ and disk amplitudes, there is coupling not only between the Lorentz-tensor structure of operators in the same left- or right-moving class but also between left and right. The latter is absent in tree amplitudes.

Correlation functions involving derivatives of $\psi$ and/or $\phi$ are treated using Wick's method in a manner completely analogous to the case of $X^{\mu}$ discussed above. Note that Eq. (II.13) applies with $\epsilon=1$ for $\phi^{i}, i=1, \ldots, 5$, and $\epsilon=-1$ for the ghost $\phi^{6}$.

Let us check that the above rules reproduce the n-point closed-string tachyon disk amplitude for the $\mathrm{D}=26$ bosonic string theory. The momenta, $k_{i}$, in the exponentials in Eq. (II.11) satisfy $\alpha^{\prime} k_{i}^{2}=4$. Multiply the right-hand-side of Eq. (II.11) by Eq. (II.14) with $z=z_{n}, w=z_{1}$, and choose $z_{1}=0, z_{n}=1$. Since the integration region requires $\left|z_{i}\right|<\exp (T)$ and $z_{n}=1$, the parameter $\mathrm{T}$ ranges from 0 to $+\infty$. Letting $\lambda=\exp (-2 T)$ and transforming $z_{i} \rightarrow 1 / z_{i}, \bar{z}_{i} \rightarrow 1 / \bar{z}_{i}$, Eq. (12) of ref. [3] is obtained. Similarly, amplitudes involving excited closed-string states may be obtained using the above rules and Eqs. (II.10, II.11, II.13, II.14).

As an example of a superstring computation, consider the massless states $|\dot{\alpha} \dot{\beta}\rangle=$ $S_{(-1 / 2)}^{L \dot{\alpha}}(0) S_{(-1 / 2)}^{R \dot{\beta}}(0)|\Omega\rangle$. The disk will generate a coupling between three of these states. 
The relevant correlation function is

$$
\begin{aligned}
&\left\langle\epsilon_{\dot{\alpha}_{3} \dot{\beta}_{3}}^{3} c^{L} S_{(-1 / 2)}^{L \dot{\alpha}_{z}}\left(z_{3}\right) c^{R} S_{(-1 / 2)}^{R \dot{\beta}_{3}}\left(\bar{z}_{3}\right) \exp \left[k_{3} \cdot\left(X^{L}\left(z_{3}\right)+X^{R}\left(\bar{z}_{3}\right)\right)\right]\right. \\
& \times \epsilon_{\dot{\alpha}_{2} \dot{\beta}_{2}}^{2} S_{(-1 / 2)}^{L \dot{\alpha}_{2}}\left(z_{2}\right) S_{(-1 / 2)}^{R \dot{\beta}_{2}}\left(\bar{z}_{2}\right) \exp \left[k_{2} \cdot\left(X^{L}\left(z_{2}\right)+X^{R}\left(\bar{z}_{2}\right)\right)\right] \\
& \times \epsilon_{\dot{\alpha}_{1} \dot{\beta}_{1}}^{1} c^{L} S_{(-1 / 2)}^{L \dot{\alpha}_{1}} c^{R}\left[\bar{\partial} X_{R}^{\mu}+\frac{\alpha^{\prime}}{8}\left(k_{1} \cdot \psi_{R}\right) \psi_{R}^{\mu}\right] \gamma_{\mu \beta}^{\dot{\beta}_{1}} S_{(+1 / 2)}^{R \beta}\left(\bar{z}_{1}\right) \\
&\left.\times \exp \left[k_{1} \cdot\left(X^{L}\left(z_{1}\right)+X^{R}\left(\bar{z}_{1}\right)\right)\right]\right\rangle,
\end{aligned}
$$

where $\epsilon_{\dot{\alpha}_{i} \dot{\beta}_{i}}^{i}$ are polarization vectors. The b-c and X-correlation functions are given in Eqs. (II.12) - (II.14). The $\psi-\phi$ ghost correlation functions are

$$
\begin{aligned}
& \left\langle S_{(-1 / 2)}^{L \dot{\alpha}_{8}}\left(z_{3}\right) S_{(-1 / 2)}^{R \dot{\beta}_{3}}\left(\bar{z}_{3}\right) S_{(-1 / 2)}^{L \dot{\alpha}_{2}}\left(z_{2}\right) S_{(-1 / 2)}^{R \dot{\beta}_{2}}\left(\bar{z}_{2}\right) S_{(-1 / 2)}^{L \dot{\alpha}_{1}}\left(z_{1}\right) S_{(+1 / 2)}^{R \beta}\left(\bar{z}_{1}\right)\right\rangle \\
= & \frac{-e^{8 T}}{\bar{z}_{2}^{2} \bar{z}_{3}^{2}}\left\langle S_{(+1 / 2)}^{L \beta}\left(\frac{e^{2 T}}{\bar{z}_{1}}\right) S_{(-1 / 2)}^{L \dot{\beta}_{2}}\left(\frac{e^{2 T}}{\bar{z}_{2}}\right) S_{(-1 / 2)}^{L \dot{\beta}_{8}}\left(\frac{e^{2 T}}{\bar{z}_{3}}\right) S_{(-1 / 2)}^{L \dot{\alpha}_{8}}\left(z_{3}\right) S_{(-1 / 2)}^{L \dot{\alpha}_{2}}\left(z_{2}\right) S_{(-1 / 2)}^{L \dot{\alpha}_{1}}\left(z_{1}\right)\right\rangle,
\end{aligned}
$$

$$
\begin{aligned}
& \left\langle S_{(-1 / 2)}^{L \dot{\alpha}_{3}}\left(z_{3}\right) S_{(-1 / 2)}^{R \dot{\beta}_{3}}\left(\bar{z}_{3}\right) S_{(-1 / 2)}^{L \dot{\alpha}_{2}}\left(z_{2}\right) S_{(-1 / 2)}^{R \dot{\beta}_{2}}\left(\bar{z}_{2}\right) S_{(-1 / 2)}^{L \dot{\alpha}_{1}}\left(z_{1}\right) \psi_{R}^{\nu} \psi_{R}^{\mu} S_{(+1 / 2)}^{R \beta}\left(\bar{z}_{1}\right)\right\rangle \\
= & \frac{e^{12 T}}{\bar{z}_{1}^{2} \bar{z}_{2}^{2} \bar{z}_{3}^{2}} \\
& \times\left\langle\psi_{L}^{\nu} \psi_{L}^{\mu} S_{(+1 / 2)}^{L \beta}\left(\frac{e^{2 T}}{\bar{z}_{1}}\right) S_{(-1 / 2)}^{L \dot{\beta}_{2}}\left(\frac{e^{2 T}}{\bar{z}_{2}}\right) S_{(-1 / 2)}^{L \dot{\beta}_{3}}\left(\frac{e^{2 t}}{\bar{z}_{3}}\right) S_{(-1 / 2)}^{L \dot{\alpha}_{3}}\left(z_{3}\right) S_{(-1 / 2)}^{L \dot{\alpha}_{2}}\left(z_{2}\right) S_{(-1 / 2)}^{L \dot{\alpha}_{1}}\left(z_{1}\right)\right\rangle,
\end{aligned}
$$

where we have used the method of images. The correlation functions in Eq. (II.21) have been computed in ref. [77]. By using the method of images, any closed-string $R P^{2}$ or disk amplitude can be related to a tree amplitude. For the superstring, all such tree amplitudes have been computed in ref. [78]. Alternatively, one can use Eqs. (II.10, II.13, II.18) above.

E. BRST Invariance

In the modern approach to strings, physical states correspond to BRST-invariant operators $O$ satisfying $\left[Q_{B R S T}, O\right]=0, O \neq\left\{Q_{B R S T}, O^{\prime}\right\}$. Operators that are BRST anticommutators generate spurious states. Their decoupling at tree level is well understood [73]. Given a correlation function involving physical operators and one spurious 
operator $\left\{Q_{B R S T}, O^{\prime}\right\}, Q_{B R S T}$ can be commuted through the other operators until it reaches the left or right $\mathrm{SL}(2, \mathrm{R})$-invariant vacuum. Since these vacua are BRST-invariant, $\left\langle\Omega\left|Q_{B R S T}=0=Q_{B R S T}\right| \Omega\right\rangle$, the correlation function is zero.

As emphasized by Friedan, Martinec and Shenker (see Section II of [73]), a similar decoupling is expected at higher loops after one integrates over moduli space. Let us analyze the situation for $R P^{2}$ and the disk $[99,100]$. The boundary operators are BRSTinvariant. However, there is a $\left(b_{0}^{R}+b_{0}^{L}\right)$ insertion. Since $Q_{B R S T}$ does not commute with the insertion, one gets $\int d T\langle B, T|\left(b_{0}^{R}+b_{0}^{L}\right) Q_{B R S T}=\int d T\langle B, T|\left(L_{0}^{R}+L_{0}^{L}\right)=\int d T \frac{d}{d T}\langle B, T|$. The violation of BRST invariance is a total derivative with respect to the parameter $T$. Under what circumstances do the boundary terms vanish? Consider the contribution at $T=+\infty$. For the $\mathrm{D}=26$ bosonic string, $\lim _{T \rightarrow \infty}\langle B, T|$ is the tree-level correlation function times $\exp (2 T)$; the latter, coming from Eq. (II.3), is the tachyon ground-state energy. Thus, the $\mathrm{D}=26$ bosonic string has problems. In fact, the original correlation function was divergent and regularization must be employed. Even with such a procedure, it appears that the decoupling of spurious states is violated and thus the perturbative formulation of the bosonic string is ill-defined. Even if a theory has no tachyon, the decoupling is unlikely to take place unless some cancellation occurs among the massless ground states. For the $\mathrm{SO}(32)$ superstring, the decoupling of spurious states probably only takes place via a cancellation of the boundary terms between the disk and $R P^{2}[3]$. 
A. Introduction

In certain string theories, transitions can take place between open and closed sectors. An example is the type I superstring. It has a massless graviton in its closed sector and massless vector bosons in its open sector. The latter provide a representation of the gauge groups $S O(N)$ or $S p(2 N)$. All these massless states will interact with each other. For SO(32), Green and Schwarz [1, 3] showed that the gravitational and the gauge anomalies cancel. They suggested that this theory is finite.

In type I superstrings, a possible source of infinities is the emission of closed strings in the presence of an open string boundary. Topologically, this is equivalent to string tubes emanating from the interior of a disk. Based on $D=26$ bosonic-string calculations, ref. [3] suggested that the infinities cancel for $S O(32)$ if the disk and $R P^{2}$ amplitudes are combined. Indeed, the combined amplitudes for graviton and dilaton emission were shown to be finite in ref. [6].

Section II discussed closed-string state emission in the presence of an open string boundary. In this Section we treat the coupling of open and closed strings, both in bosonic and in supersymmetric string theories. This corresponds to placing open-string vertex operators at the boundary of the disk and closed-string vertex operators on its interior. Thus, with a few modifications, the results of Section II can be adapted to mixed openand closed-string amplitudes.

These mixed amplitudes have, for the most part, been little analyzed. Recently, Shapiro and Thorn have constructed a vertex operator that couples an open bosonic string to a closed bosonic string $[101]$; see also $[19,21]$. As an application, they compute the decay of one closed bosonic string into n open-string tachyons. 
B. Rules

(1) For the left state use the boundary operator $\langle B, 0|$ at $T=0$, i.e, a unit disk. For the right state use $|\Omega\rangle$.

(2) For closed strings, use the standard closed-string vertex operators. For open strings, a prescription is needed for converting them into conformal fields on the disk, i.e., leftor right-moving closed-string operators. Setting aside $X_{\text {open }}(\sigma, \tau)$, the prescription is as follows. If $\int d z O(z)$ is an open string vertex operator, replace it by $\int d z O_{L}(z)$ where the integral now ranges around the boundary of the unit disk. For a $c(z) O(z)$ vertex, replace it by $c_{L}(z) O_{L}(z)$. For exponentials of $X_{\text {open }}(\sigma, \tau)$ there is a subtlety: $\exp \left(k \cdot X_{\text {open }}(\sigma, \tau)\right) \rightarrow$ $\exp \left(k \cdot 2 X_{e q}^{L}(z)\right)$. In short, open-string operators are expressed in terms of the holomorphic variable $z$ and then replaced by their left-moving closed counterparts. One closed $c^{L} c^{R_{-}}$ type vertex and one open c-type vertex are to be used. The total $\phi$-ghost number must sum to $\mathbf{- 2}$.

(3) Evaluate the correlation functions using the formulae and methods described below.

(4) For a closed-string variable $z_{i}$, the integration is $\int_{\left|z_{i}\right|<1} d^{2} z_{i}$. For $z_{i}$ associated with an open vertex, it is $\oint_{\left|x_{i}\right|=1} d z_{i}$. The open-string operators appear to the left of all closed-string operators because $|z|$ for an open string is greater than $|z|$ for a closed string.

Note that there is no parameter $T$. The disk has radius fixed to one, so that formulae in Section II are applicable with $T$ set to zero.

Rule (2) means that $\exp (\lambda \cdot \phi)(z) \rightarrow \exp \left(\lambda \cdot \phi^{L}\right)(z), \partial X^{\mu}(z) \rightarrow \partial X_{L}^{\mu}(z), c(z) \rightarrow c^{L}(z)$, $b(z) \rightarrow b^{L}(z)$, etc. Integrations such as $\int d z$ become $\oint_{|z|=1} d z$.

The prescription for $\exp \left(k \cdot X_{\text {open }}(\sigma, \tau)\right)$ and the factor of 2 in the exponent in rule (2) require some, explanation. From a functional-integral viewpoint, there is only one $X(\sigma, \tau)$ operator, so $X_{\text {open }}(\sigma, \tau)$ should become $X_{\text {closed }}(\sigma, \tau)$. Using Eqs. (I.2) and (I.4), one sees that this corresponds to $X(z) / 2 \rightarrow X_{e q}^{L}(z)$ and $X(\bar{z}) / 2 \rightarrow X_{e q}^{R}(\bar{z})$. To gain additional insight, begin with the open-string operators defined on the upper half plane and perform 
the conformal transformations

$$
\begin{aligned}
& z=i\left(\frac{1-w}{1+w}\right), \\
& w=\left(\frac{1+i z}{1-i z}\right),
\end{aligned}
$$

where $z$ is the upper-half-plane variable and $w$ is the disk variable. For example, on the real axis, tachyon operators such as $\int d x \exp \left(k \cdot X_{\text {open }}(\sigma, \tau)\right)$ become $\int d z \exp [k \cdot 2(X(z) / 2)]$. Here, $z=x+i y$. The factor of two now follows from the equivalence $X(z) / 2 \leftrightarrow X_{e q}^{L}(z)$. An alternative procedure replaces $\int d x \exp \left(k \cdot X_{\text {open }}(\sigma, \tau)\right)$ by $\int_{0}^{2 \pi} d \theta \exp \left[k \cdot X_{\text {closed }}(z, \bar{z})\right]$, where $z=\exp (i \theta)$, the only subtlety being that $d x \rightarrow d \theta=-i d z / z$.

An open-string operator that has been converted to a left-moving disk operator can instead be replaced by a right-moving operator via Eq. (II.9): $\langle B, 0| \oint_{|z|=1} d z O_{L}(z)=$ $\langle B, 0| \oint_{|z|=1} d z\left(\frac{-1}{z}\right) O_{R}\left(\frac{1}{z}\right)=\langle B, 0| \oint_{|z|=1} d \bar{z} O_{R}(\bar{z})$, where we have used that $O$ has conformal weight one. Since this can be done with each open-string operator, the procedure in rule (2) is independent of whether the operators are converted to left- or right-moving disk operators. Technically speaking, the open-string operators $O_{i}^{L}\left(z_{i}\right), i=1, \ldots, m$, should be integrated around concentric circles $\left|z_{i}\right|=1-(n-i) \epsilon$ so that an ordering of the operators is achieved. In the end, the limit $\epsilon \rightarrow 0$ can be taken.

C. Correlation Functions

Correlation functions can be computed using the results in Section II with $T \rightarrow 0$. For example,

$$
\begin{aligned}
& \left\langle B, 0\left|\exp \left[2 p_{m} \cdot X_{L}\left(w_{m}\right)\right] \ldots \exp \left[2 p_{1} \cdot X_{L}\left(w_{1}\right)\right] \exp \left[k_{n} \cdot X\left(z_{n}, \bar{z}_{n}\right)\right] \ldots \exp \left[k_{1} \cdot X\left(z_{1}, \bar{z}_{1}\right)\right]\right| \Omega\right\rangle \\
& =\prod_{\ell>k}\left(w_{\ell}-w_{k}\right)^{2 \alpha^{\prime} p_{\ell} \cdot p_{k}} \times \prod_{\ell, i}\left[\left(w_{\ell}-z_{i}\right)\left(1-w_{\ell} \bar{z}_{i}\right)\right]^{\alpha^{\prime} p_{\ell} \cdot k_{i}}, \\
& \quad \times[R H S \text { of } E q . \text { (II.11) at } T=0] \times \delta^{D}\left(\sum_{\ell=1}^{m} p_{\ell}+\sum_{i=1}^{n} k_{i}\right)
\end{aligned}
$$


Correlation functions involving $\partial X_{L}^{\mu}(z)$ and $\bar{\partial} X_{R}^{\mu}(\bar{z})$ are obtained using Wick's rules in Eq. (II.13) with $T \rightarrow 0$ and $\epsilon \rightarrow \alpha^{\prime} / 2$.

The only relevant b-c expectation is

$$
\begin{aligned}
& \left\langle B, 0\left|c^{L}(w) c^{R}(\bar{z}) c^{L}(z)\right| \Omega\right\rangle \\
& \quad=-(1-w \bar{z})(w-z)(1-z \bar{z})
\end{aligned}
$$

because only three c's occur according to rule (2).

Similarly, $\psi-\phi$ correlations can be computed in their exponential representation (see Eq. (I.1)):

$$
\begin{aligned}
& \left\langle B, 0\left|O^{\lambda_{m}}\left(w_{m}\right) \ldots O^{\lambda_{1}}\left(w_{1}\right) O^{\lambda_{n}^{L} \lambda_{n}^{R}}\left(z_{n}, \bar{z}_{n}\right) \ldots O^{\lambda_{1}^{L} \lambda_{1}^{R}}\left(z_{1}, \bar{z}_{1}\right)\right| \Omega\right\rangle \\
& =\prod_{\ell>k}\left\{\exp \left[i \pi \lambda_{\ell} \cdot M \lambda_{k}\right]\left(w_{\ell}-w_{k}\right)^{\left\langle\lambda_{\ell} \cdot \lambda_{k}\right\rangle}\right\} \\
& \quad \times \prod_{\ell, i}\left\{\exp \left[i \pi \lambda_{\ell} \cdot M\left(\lambda_{i}^{L}+\lambda_{i}^{R}\right)\right]\left(w_{\ell}-z_{i}\right)^{\left\langle\lambda_{\ell} \cdot \lambda_{i}^{L}\right\rangle}\left(1-w_{\ell} \bar{z}_{i}\right)^{\left\langle\lambda_{\ell} \cdot \lambda_{i}^{R}\right\rangle}\right\} \\
& \quad \times[R H S \text { of Eq. (II.18) at } T=0] \times \delta_{\sum_{\ell=1}^{m} \lambda_{\ell}+\sum_{i=1}^{n}\left(\lambda_{i}^{L}+\lambda_{i}^{R}\right),(0 ;-2)},
\end{aligned}
$$

where the first $m$ operators and the variables $w_{1}, \ldots, w_{m}$ are associated with open strings. Terms with derivatives are calculated from Eq. (II.13).

Let us check that the above rules generate correct amplitudes.

One can obtain pure open-string amplitudes by using only open-string operators, three of which are c-type vertices. The relevant b-c correlation function is

$$
\left\langle B, 0\left|c^{L}\left(z_{3}\right) c^{L}\left(z_{2}\right) c^{L}\left(z_{1}\right)\right| \Omega\right\rangle=-\left(z_{3}-z_{2}\right)\left(z_{2}-z_{1}\right)\left(z_{3}-z_{1}\right)
$$

From (II.2) and (III.5) the $n$ open-string tachyon amplitude is 


$$
\begin{aligned}
& \prod_{j=1}^{m} \oint_{\left|w_{i}\right|=1} d w_{i} \prod_{j>i}\left(w_{j}-w_{i}\right)^{2 \alpha^{\prime} p_{j} \cdot p_{i}} \frac{\left(w_{n}-w_{k}\right)\left(w_{n}-w_{\ell}\right)\left(w_{\ell}-w_{k}\right)}{\left[d w_{n} d w_{\ell} d w_{k}\right]} \\
& =\prod_{j=1}^{m} \int_{0}^{2 \pi} d \theta_{i} \prod_{j>i}\left[4 \sin ^{2}\left(\theta_{j}-\theta_{i}\right)\right]^{\alpha^{\prime} p_{j} \cdot p_{i}} \\
& \quad \times \frac{8\left[\sin ^{2}\left(\theta_{n}-\theta_{k}\right) \sin ^{2}\left(\theta_{n}-\theta_{\ell}\right) \sin ^{2}\left(\theta_{\ell}-\theta_{k}\right)\right]^{1 / 2}}{\left[d \theta_{n} d \theta_{\ell} d \theta_{k}\right]}
\end{aligned}
$$

where $\left[d w_{n} d w_{\ell} d w_{k}\right]^{-1}$ and $\left[d \theta_{n} d \theta_{\ell} d \theta_{k}\right]^{-1}$ symbolically indicate that these integrations are to be omitted. The conditions $\alpha^{\prime} p_{i}^{2}=1$ and $w_{i}=\exp \left(i \theta_{i}\right)$ have been used in (III.6). Equation (III.6) agrees with the standard result.

The amplitude for $n$ open-string tachyons and one closed-string tachyon has been computed in ref. [101]. From our rules, the relevant correlation function is

$$
\begin{aligned}
& \prod_{\ell=2}^{m} \int d w_{i}\langle B, 0| \exp \left[2 p_{m} \cdot X^{L}\left(w_{m}\right)\right] \ldots \exp \left[2 p_{2} \cdot X^{L}\left(w_{2}\right)\right] \\
& \quad \times c_{L}\left(w_{1}\right) \exp \left[2 p_{1} \cdot X^{L}\left(w_{1}\right)\right] c_{L}(z) c_{R}(\bar{z}) \exp \left[k \cdot X_{\text {closed }}(z, \bar{z})\right]|\Omega\rangle \\
& \quad=\prod_{\ell=2}^{m} \int_{0}^{2 \pi} \frac{d \theta_{i}}{2} \prod_{m \geq j>i \geq 1}\left(\sin ^{2} \frac{\theta_{j} \cdot \theta_{i}}{2}\right)^{\alpha^{\prime} p_{j} \cdot p_{i}},
\end{aligned}
$$

where we have fixed $w_{1}=1$ and $z=\bar{z}=0$ and used $\alpha^{\prime} p_{i}^{2}=1, \alpha^{\prime} k^{2}=4$. Equation (III.7) is the correct result [101]. The substitution of a massless closed-string state for the closedstring tachyon corresponds to substituting $\partial X_{L}^{\mu}(z) \partial X_{R}^{\nu}(\bar{z}) \exp [k \cdot X(z, \bar{z})]$ for $\exp [k \cdot X(z, \bar{z})]$ in Eq. (III.7) and constraining $\alpha^{\prime} k^{2}=0$. According to Eq. (II.13), we get the following extra factors:

$$
\begin{aligned}
\partial X_{L}^{\mu}(z) \bar{\partial} X_{R}^{\nu}(\bar{z}) & \rightarrow-\frac{\alpha^{\prime}}{2} \eta^{\mu \nu} \frac{1}{(1-z \bar{z})^{2}} \rightarrow-\frac{\alpha^{\prime}}{2} \eta^{\mu \nu}, \quad z=\bar{z}=0, \\
e^{2 p_{j} \cdot X^{L}}\left(w_{j}\right) e^{2 p_{i} \cdot X^{L}}\left(w_{i}\right) \partial X_{L}^{\mu}(z) \bar{\partial} X_{R}^{\nu}(\bar{z}) & \rightarrow\left(\frac{\alpha^{\prime}}{2}\right)^{2} 4 p_{j}^{\mu} p_{i}^{\nu} \frac{1}{\left(z-w_{j}\right)}\left(\frac{-w_{i}}{1-w_{i} \bar{z}}\right) \\
\underbrace{.} & \rightarrow\left(\alpha^{\prime}\right)^{2} p_{j}^{\mu} p_{i}^{\nu} e^{i\left(\theta_{i}-\theta_{j}\right)}, z=\bar{z}=0,
\end{aligned}
$$

where $w_{i}=\exp \left(i \theta_{i}\right)$. The integrand in Eq. (III.7) thus gets multiplied by 


$$
-\left(\frac{\alpha^{\prime}}{2}\right)\left[\eta^{\mu \nu}-2 \alpha^{\prime} \sum_{j, i} p_{j}^{\mu} p_{i}^{\nu} e^{i\left(\theta_{i}-\theta_{j}\right)}\right] .
$$

This again agrees with ref. [101].

Finally, let us compute a superstring amplitude involving the graviton, two massless open-string fermions and an open-string massless vector. The relevant correlation function is

$$
\begin{aligned}
& \left\langle B, 0\left|V_{(0)}^{L c}\left(z_{4}\right) V_{(+1 / 2)}^{L} V_{(-1 / 2)}^{L} \int_{\mu \nu} c^{L} \psi_{(-1)}^{L \mu}\left(z_{1}\right) c^{R} \psi_{(-1)}^{R \nu}\left(\bar{z}_{1}\right) \exp \left[k \cdot X_{\text {closed }}(z, \bar{z})\right]\right| \Omega\right\rangle \\
& =\epsilon_{\mu \nu}\langle\Omega| c^{L}\left(\frac{1}{\bar{z}_{1}}\right) \psi_{(-1)}^{L \nu}\left(\frac{1}{\bar{z}_{1}}\right) \exp \left[k \cdot X^{L}\left(\frac{1}{\bar{z}_{1}}\right)\right] \\
& \quad \times V_{(0)}^{L c}\left(z_{4}\right) V_{(+1 / 2)}^{L} \int_{(-1 / 2)}^{L} c^{L} \psi_{(-1)}^{L \mu}\left(z_{1}\right) \exp \left[k \cdot X^{L}\left(z_{1}\right)\right]|\Omega\rangle,
\end{aligned}
$$

where the method of images has been used. The vertex operators in Eq. (III.10) are given in Eq. (IV.1) of ref. [78]. Points 1, 2, 3 and 4, respectively, are associated with the massless vector, the first massless fermion, the second massless fermion, and the graviton. Eq. (III.10) is derived in Eqs. (IV.10-TV.18) of [78]. 


\section{ANNULUS AND MÖBIUS-STRIP AMPLITUDES}

\section{A. Introduction}

Take a strip of width $\pi$ and length $T$ and join opposite ends. The result is an annulus; see Figure 1a. Twisting one end before joining gives the Möbius strip. One-loop corrections to open-string scattering amplitudes are derived by attaching vertex operators at the boundaries. The resulting functional integrals lead to conformal field theories on the annulus and Möbius strip.

This Section provides methods for calculating amplitudes involving these two topologies. The effects of ghosts are incorporated, and spin fields are defined via bosonization.

One-loop amplitudes for tachyons and massless states have previously been computed. These amplitudes are given in ref. [27] in the light-cone gauge. Results in this Section differ from [27] in that general amplitudes are obtained and that a covariant approach is used. The modern approach based on conformal methods facilitates the computations.

The variable $z$ of Eq. (I.7) is used. Operators then have mode expansions in powers of $z$ [73]. See [78] for our conventions. In terms of $z$, Figure 1a becomes Figure 1b, the half-annulus with interior and exterior semicircles identified.

We first present rules for calculating the amplitudes. Next, we supply a complete list of propagators and methods for deriving the correlation functions.

B. Rules

(1) The amplitude is

$$
\int d T\left\langle b_{0} V_{n} \ldots V_{1}\right\rangle
$$

where n-1 integral-type vertices and one c-type vertex are used. For the superstring, the total $\phi$-ghost charge must sum to zero. The vertex operators are those used at tree level. 
(2) The integration region for the parameter $T$ is 0 to $\infty$. For the $j^{\text {th }}$ integral vertex, $z_{j}$ is integrated from -1 to $-\exp (T)$ and from 1 to $\exp (T)$ provided all vertex operators are distinct; otherwise, an ordering must be introduced.

(3) Evaluate correlation functions using the formulae and methods in subsection $\mathrm{C}$ below.

For (2), set $\rho_{j}=\ln z_{j}=\tau_{j}+i \sigma_{j}$. Then, the region from 1 to $\exp (T)$ corresponds to $\sigma_{j}=0,0 \leq \tau_{j} \leq T$. The region from -1 to $-\exp (T)$ corresponds to $\sigma_{j}=\pi, 0 \leq \tau_{j} \leq T$, i.e., the upper and lower boundaries of Figure 1a.

An amplitude is planar if all the $z_{i}$ are in one of the two intervals mentioned in rule (2). Otherwise, it is non-planar and contains closed strings as intermediate states [27].

The single $b_{0}$ insertion and the flat measure over $T$ is related to BRST invariance. This is discussed in subsection IV.E.

C. Correlation Functions

The evaluation of a correlation function naturally separates into the contribution from oscillators and the contribution from zero modes such as $[\phi]_{0}$ and $[\partial \phi]_{0}$ for any field $\phi$. Here, $[\phi]_{0}$ and $[\partial \phi]_{0}$ are the center-of-mass and the momentum coordinates of $\phi$. The trace for $X^{\mu}$ involves an integral over the momentum, whereas a sum over a lattice appears for fields $\phi$ associated with a bosonization procedure or a toroidal compactification.

In what follows, it is useful to define a universal function related to the oscillator contribution:

$$
\begin{aligned}
g_{o e c}(z, w, T) & =\ln \left(1-\frac{w}{z}\right)+\sum_{m=1}^{\infty} \ln \left[\frac{\left(1-\frac{z}{w} e^{-m T}\right)\left(1-\frac{w}{x} e^{-m T}\right)}{\left(1-e^{-m T}\right)^{2}}\right] \\
& =\ln \left[2 \pi i \sqrt{\frac{w}{z}} \frac{\Theta\left[\frac{\frac{1}{2}}{\frac{1}{2}}\right]\left(\nu_{x}-\nu_{w} \mid \tau\right)}{\Theta^{\prime}\left[\frac{\frac{\alpha}{z}}{2}\right](0 \mid \tau)}\right]
\end{aligned}
$$

where $|z|>|w|$ and 


$$
\begin{aligned}
\nu_{z} & =\frac{1}{2 \pi i} \ln z, \quad \nu_{w}=\frac{1}{2 \pi i} \ln w, \\
\tau & =\frac{i T}{2 \pi}, \\
\Theta^{\prime}(0, \tau) & =\left.\frac{d}{d \nu} \Theta(\nu \mid \tau)\right|_{\nu=0} .
\end{aligned}
$$

The definition of the theta function [113] is

$$
\begin{aligned}
\Theta\left[\begin{array}{l}
\theta \\
\phi
\end{array}\right]\left(\nu, \frac{i T}{2 \pi}\right) & =\sum_{n=-\infty}^{\infty} \exp \left[-(n+\theta)^{2} \frac{T}{2}+2 \pi i(n+\theta)(\nu+\phi)\right] \\
& =e^{-\frac{\pi}{2} \theta^{2}} \exp [2 \pi i \theta(\phi+\nu)] \prod_{n=1}^{\infty}\left(1-e^{-n T}\right) \\
& \times \prod_{n=1}^{\infty}\left(1+e^{-T(n+\theta-1 / 2)} e^{2 \pi i(\phi+\nu)}\right)\left(1+e^{-T(n+\theta-1 / 2)} e^{-2 \pi i(\phi+\nu)}\right)
\end{aligned}
$$

where the latter equality is the product representation.

Correlation functions for $X^{\mu}$ are evaluated using Wick's theorem and the following propagator:

$$
\begin{aligned}
g_{X}(z, w, T) & =\frac{1}{2 \alpha^{\prime}}\langle X(z) X(w)\rangle_{T} \\
& =\left\{1 / 4\left[g_{o s c}(z, w, T)+g_{o s c}(\bar{z}, w, T)+g_{o s c}(z, \bar{w}, T)\right.\right. \\
& \left.\left.+g_{o s c}(\bar{z}, \bar{w}, T)\right]+g_{\text {zero }}(z, w, T)\right\}
\end{aligned}
$$

The contribution $g_{z e r o}(z, w, T)$, from zero modes is

$$
\begin{aligned}
g_{z e r o}(z, w, T) & =\ln |z|-\frac{1}{2 \operatorname{Re} T}\left(\ln \left|\frac{z}{w}\right|\right)^{2} \\
& =1 / 2(\ln z+\ln \bar{z})-\frac{1}{8 R e T}(\ln z+\ln \bar{z}-\ln w-\ln \bar{w})^{2}
\end{aligned}
$$

where $|z|>|w|$. Even though $T$ is real at this stage, below we need results for complex T. $R e T$ stands for the real part of T. Eq. (IV.4) is obtained by decomposing $X^{\mu}$ into oscillators and using the results of Appendix $\mathbf{A}$. 
As a check, the $T \rightarrow \infty$ limit of an annulus correlation should be the correlation for the infinite strip, i.e., the tree-level result. In taking this limit, first scale the $z_{i}$ by $\exp (-T / 2)$ so that they remain in the middle of the strip. Then, Eq. (IV.4) reduces to the tree-level propagator for $T \rightarrow \infty$. For other topologies and correlation functions, we made frequent use of this check.

Actually, Eq. (IV.4) is not the true $X^{\mu}$ propagator but an effective one. The operator $X^{\mu}$ only appears in exponentials. Since exponentials on the annulus have self-energy contributions that are different from those at tree level, the normal-ordering procedure used at tree level does not eliminate the self-energy contributions at the one-loop level. These extra effects produce the factor $-\sum_{m=1}^{\infty} \ln \left(1-e^{-m T}\right)^{2}$ in Eq. (IV.1). This term, being independent of $z$ and $w$, disappears for derivatives of $X^{\mu}$. With the effective propagator, an $X^{\mu}$ should only be contracted with an $X^{\mu}$ in another exponential. For the reader interested in working through this technicality, the following is a useful identity:

$$
: e^{k \cdot X}(z):=: e^{k \cdot X}: \exp \left[-\alpha^{\prime} k^{2} \ln |z|\right]\left(\prod_{m=1}^{\infty}\left(1-e^{-m T}\right)\right)^{2 \alpha^{\prime} k^{2}},
$$

where :: denotes normal ordering on the half-plane and $\because:$ denotes normal ordering on the annulus. To show the equivalence of these two procedures in a correlation function of exponentials, the constraint $\sum_{\ell} k_{\ell}=0$ must be used.

It is illustrative to derive the chiral oscillator propagator, $g_{o s c}$, from the method of images discussed in the Introduction. The oscillator contribution to the propagator on the infinitely long strip is $\left\langle\phi_{\text {osc }}\left(z_{2}\right) \phi_{\text {osc }}\left(z_{1}\right)\right\rangle=\ln \left(1-z_{\langle} / z_{\rangle}\right)$where $z_{\langle}$is $z_{1}$ or $z_{2}$ depending on whether $\left|z_{1}\right|<\left|z_{2}\right|$ or $\left|z_{2}\right|<\left|z_{1}\right|$. To obtain $g_{o s c}$ for a finite strip of length $T$, introduce mirror charges at $z_{1} \exp (m T)$ for $m= \pm 1, \pm 2, \ldots$. This makes $g_{\text {osc }}$ periodic in $\tau$ with period $T$. Summing the contributions yields $\sum_{m=-\infty}^{\infty}\left\langle\phi_{\text {osc }}\left(z_{2}\right) \phi_{\text {osc }}\left(z_{1} e^{m T}\right)\right\rangle=$ $\ln \left(1-\frac{z_{1}}{z_{2}}\right)+\sum_{m=1}^{\infty} \ln \left(1-z_{1} e^{-m T} / z_{2}\right)\left(1-z_{2} e^{-m T} / z_{1}\right)$ for $\left|z_{2}\right|>\left|z_{1}\right|$. This is Eq. (IV.1) without the self-energy factor, $\sum_{m=1}^{\infty} \ln \left(1-e^{-m T}\right)^{-2}$.

From Eq. (IV.4) it follows that 


$$
T\left\langle e^{k_{n} \cdot X}\left(z_{n}\right) \ldots e^{k_{1} \cdot X}\left(z_{1}\right)\right\rangle_{X}=\prod_{j>i} \exp \left[g_{X}\left(z_{j}, z_{i}, T\right) 2 \alpha^{\prime} k_{j} \cdot k_{i}\right]_{T}\langle 1\rangle_{X}
$$

where

$$
r\langle 1\rangle_{X}=[f(T)]^{-D}\left(\frac{1}{4 \pi \alpha^{\prime} \operatorname{ReT}}\right)^{D / 2}
$$

with

$$
f(T)=\prod_{m=1}^{\infty}\left(1-e^{-m T}\right)
$$

$T\langle 1\rangle_{X}$ is the partition function $\operatorname{Tr} \exp (-T H)$ associated with the $X^{\mu}$ degrees of freedom. The $f(T)$ term, which comes from the oscillators, is obtained from Eq. (A.7). The 1/T factors come from the zero modes, Eq. (A.9).

Derivatives of $X^{\mu}$ in a correlation function are removed using Wick's theorem. Associate the following factors with contractions:

$$
\begin{aligned}
& \partial X^{\mu}(z) e^{k \cdot X}(w) \rightarrow 2 \alpha^{\prime} k^{\mu}\left[2 \frac{\partial}{\partial z} g_{X}(z, w, T)\right] \\
& \partial X^{\mu}(z) \partial X^{\nu}(w) \rightarrow 2 \alpha^{\prime} \eta^{\mu \nu}\left[4 \frac{\partial}{\partial z} \frac{\partial}{\partial w} g_{X}(z, w, T)\right]
\end{aligned}
$$

Eq. (IV.10) follows from Eq. (IV.4) by differentiation, treating $z$ and $\bar{z}$ as independent variables. Higher-derivative terms are treated by further differentiation. The extra factors of two arise from Eq. (I.2).

Since one $b_{0}$ appears and one c-type vertex is used, the relevant b-c correlation function is

$$
T\left\langle b_{0} c(z)\right\rangle_{b c}=z e^{R e T}[f(T)]^{2}
$$

The factor of $\exp (T)$ is due to the tachyon. In the modern approach [73], it is associated with the state $c_{1}|\Omega\rangle$. Note that $T_{T}\langle 1\rangle_{b c}=0$ due to the $c_{0}$ mode: for every bosonic state there 
is a degenerate partner obtained by applying $c_{0}$ or $b_{0}$. Thus, the $b_{0}$ insertion eliminates the zero-mode degeneracy.

As an example, consider the $n$ open-string tachyon amplitude. Without loss of generality, the c-type vertex can be taken as the first vertex operator and the corresponding $z_{1}$ can be fixed to one. Multiplying Eq. (IV.7) by Eq. (IV.11) gives the amplitude. The change of variables

$$
\begin{aligned}
w & =e^{-T}, \\
\rho_{i} & =e^{-T} z_{n} / z_{i} \\
c_{j i} & =\rho_{j} / \rho_{i}
\end{aligned}
$$

leads to the standard result, Eq. (5.15) of ref. [27].

Next, we bosonize the $\psi$ field; see Section II of [78] for conventions. Let us organize the results into a framework that covers the general bosonization scheme.

Consider $\mathrm{N}$ bosons, $\phi^{i}, i=1, \ldots, N$, associated with the bosonization of $2 \mathrm{~N}$ fermions. In principle, there can be background charges, $Q_{i}$, for the $i^{\text {th }}$ boson. This situation includes: (a) the b-c system with $c=\exp (\sigma), b=\exp (-\sigma)$ and $Q=-3$; (b) the $\xi-\eta$ system with $\xi=\exp (\chi), \eta=\exp (-\chi)$ and $Q=-1 ;$ (c) $\psi$ bosonization with $\phi^{i}, i=1, \ldots, 5$, and $Q=0$; (d) bosons associated with a toroidal compactification scheme. Case (d) covers heterotic strings $[2,103,114-119]$ for which there are at least 16 bosons with $Q_{i}=0$.

For operators of the form in Eq. (I.1), again separate the contribution into an oscillator part and a zero-mode part. Arrange the eigenvectors of $\left[\partial \phi^{i}\right]_{0}$ into a vector $|q|:\left[\partial \phi^{i}\right]_{0}|q\rangle=$ $q^{i}|q\rangle$. In applications, these vectors may span the weight lattice of an algebra. Integer entries yield states in the NS sector; half-integer ones yield states in the $\mathrm{R}$ sector. For the zero-mode contribution, a trace over these vectors is taken. It is convenient to weight the trace by an extra phase $\exp \left(i \pi \alpha_{i} q^{i}\right)$ with the $\alpha_{i}$ equal to +1 or 0 , so that GSO projections can be implemented. Thus, instead of summing only over GSO-accepted states, sum over all states weighting GSO-accepted states by 1 and GSO-rejected states by a phase $\exp (i \pi \alpha)$. 
Then, average over $\alpha=0$ and 1 to implement the GSO projection. The phase $\exp (i \pi \alpha)$ is the spin structure in the $\tau$ direction. Summing over spin structures is equivalent to the GSO projection [103].

For operators of the form in Eq. (I.1) we get:

$$
T\left\langle O^{\lambda_{n}}\left(z_{n}\right) \ldots O^{\lambda_{1}}\left(z_{1}\right)\right\rangle_{\beta, t}=\{\text { oscillator factor }\} \times\{\text { lattice factor }\}
$$

where

$$
\begin{aligned}
& \{\text { oscillator factor }\}=[f(T)]^{-N} \prod_{m>\ell} \exp \left[\lambda_{m} \cdot \lambda_{\ell} g_{o o c}\left(z_{m}, z_{\ell}, T\right)\right] \\
& \{\text { lattice factor }\}=\prod_{i=1}^{N} \Lambda\left(\beta, \alpha_{i}, Q_{i}, T,\left\{z_{\ell}\right\}\right) \times \prod_{m>\ell} \exp \left[\lambda_{m} \cdot \lambda_{\ell} \ln z_{m}+i \pi \lambda_{m} \cdot M \lambda_{\ell}\right] \\
& \times \sum_{\sum_{\ell=1}^{n} \lambda_{l}^{i}, 0} \\
& \Lambda\left(\beta, \alpha_{i}, Q_{i}, T,\left\{z_{\ell}\right\}\right)=\sum_{q^{i} \in Z+\beta} W\left(q^{i}, Q_{i}, \alpha_{i}, \lambda_{\ell}^{i},\left\{z_{\ell}\right\}\right) \\
& =\exp \left[\frac{Q_{i}^{2}}{8} T+\frac{i \pi Q_{i} \alpha_{i}}{2}-\frac{Q_{i}}{2} \sum_{\ell} \lambda_{\ell}^{i} \ln z_{\ell}\right] \\
& \times \Theta\left[\begin{array}{c}
\beta+\frac{1}{2} Q_{i} \\
\frac{1}{2} \alpha_{i}
\end{array}\right]\left(\frac{1}{2 \pi i} \sum_{\ell} \lambda_{\ell}^{i} \ln z_{\ell} \mid \frac{i T}{2 \pi}\right) \\
& W\left(q^{i}, Q_{i}, \alpha_{i}, \lambda_{\ell}^{i},\left\{z_{\ell}\right\}\right)=\exp \left[-\frac{1}{2} q^{i}\left(q^{i}+Q_{i}\right) T\right] \exp \left[i \pi \alpha_{i} q^{i}\right] \exp \left[q^{i} \sum_{\ell} \lambda_{\ell}^{i} \ln z_{\ell}\right]
\end{aligned}
$$

The first factor in Eq. (IV.14) is the partition function for the oscillators (Eq. (A.7)). The role of the $\alpha_{i}$ is described above, $T$ is the length of the annulus, the function $g_{o s c}$ is given in Eq. (IV.1), and the theta-function equivalence follows from Eq. (IV.3). Correlation functions are non-zero only if they satisfy the appropriate background-charge constraint 
for the $i^{\text {th }}$ boson. The $q^{i}$ in Eq. (IV.16) are the eigenvalues of $\left[\partial \phi^{i}\right]_{0}$ in the lattice trace. Finally, in Eqs. (IV.13-16), $\beta=0$ and $\beta=\frac{1}{2}$ correspond, respectively, to the NS and $R$ sectors, while $\exp (2 \pi i \beta)$ is the spin structure in the $\sigma$ direction.

The term $\prod_{m>\ell} \exp \left(i \pi \lambda_{m} \cdot M \lambda_{\ell}\right)$, generated from the $c_{\lambda}$ in Eq. (I.1), yields the Lorentz-tensor structure in an amplitude. The effect of the $c_{\lambda}$ is calculated by moving them to the right and collecting their action on $|q\rangle$. In the process, the product factor is generated. At tree-level, $c_{\lambda}|\Omega\rangle=|\Omega\rangle$ and these operators disappear. Here, $c_{\lambda}|\mathbf{q}\rangle \neq|\mathbf{q}\rangle$. Instead, a term $\exp \left[i \pi \sum_{\ell} \lambda_{l} M[\partial \phi]_{0}\right]$ appears. However, since $\sum_{\ell} \lambda_{l}=0$ due to the background-charge constraint in Eq. (IV.15), $\exp \left[i \pi \sum_{\ell} \lambda_{\ell} M[\partial \phi]_{0}\right]=1$.

Let us illustrate the use of the $\alpha_{i}$ to implement a GSO projection for an so(8) internalsymmetry algebra. There are four sectors, (o), (v), (s) and (c), each of which is associated with a weight vector: $w_{o}(0)=(0,0,0,0), w_{v}(0)=(1,0,0,0), w_{s}(0)=\left(\frac{1}{2}, \frac{1}{2}, \frac{1}{2}, \frac{1}{2}\right)$ and $w_{c}(0)=\left(\frac{1}{2}, \frac{1}{2}, \frac{1}{2},-\frac{1}{2}\right)$. The subset of the weight lattice for each sector is obtained from the appropriate weight vector by repeatedly adding $\pm e_{i} \pm e_{j}$, where $e_{i}$ is a unit vector in the $i^{\text {th }}$ direction. The variable $\beta$, introduced above, is 0 for the (o) and (v) sectors and $\frac{1}{2}$ for (s) and (c). Suppose one wants to project onto the (o) and (s) sectors. Choose $\alpha_{1}=-(1+\alpha)$ and $\alpha_{i}=(1-\alpha)$ for $i=2,3,4$, where $\alpha=0$ or 1 . Then, the sectors (o), (v), (s) and (c) are weighted by $1,-(-)^{\alpha},(-1)$, and $(-1)^{\alpha}$ respectively. By averaging over $\alpha=1$ and 0 , the sectors (o) and (s) are selected and a graded trace is generated.

Let us next discuss how to treat derivative terms for this system of bosons. Although the weight factor is quadratic in $\mathbf{q}$, the $q^{i}$ degrees of freedom are not continuous variables integrated from $-\infty$ to $+\infty$. Therefore, Wick's theorem cannot be used. Instead, one must first fix $|q\rangle$, then use Wick's theorem, and finally sum over the discrete values $q_{i}$. In addition, the zero modes can act on $|q\rangle$, and hence contractions between operators and |q) take place. The rules for a $\partial \phi^{i}(z)$ term are as follows. Draw all possible contractions between $\partial \phi^{i}(z)$ and other operators. Include a contraction with $|q\rangle$. Associate the following factors with these contractions: 


$$
\begin{aligned}
& \partial \underline{\phi}^{i}(z) e^{\lambda \cdot \phi}(w) \rightarrow\left[\lambda^{i} \frac{\partial}{\partial z} g_{o s c}(z, w, T)+\frac{\lambda^{i}}{z}\right] \\
& \partial \phi^{i}(z)|q\rangle \rightarrow \frac{q^{i}}{z} \\
& \partial \phi^{i}(z) \partial \phi^{j}(w) \rightarrow \delta^{i j} \frac{\partial}{\partial z} \frac{\partial}{\partial w} g_{o s c}(z, w, T)
\end{aligned}
$$

Finally, sum over the $q^{i}$ with weight factor $W\left(q_{i}, Q_{i}, \alpha_{i}, \lambda_{\ell}, z_{\ell}\right) \times$ (oscillator factor); see Eqs. (IV.14) and (IV.16).

This discussion is valid for bosonized systems with $\epsilon=1$ [73]. This is sufficient for all superstring fields except the $\phi$-ghost, which has $\epsilon=-1$ and $Q=2$ [73]. The $\phi$-ghost is difficult to handle for the following reason. The correct set of states for the trace is generated by the non-negative modes of $\beta=\partial \xi \exp (-\phi)$ and $\gamma=\exp (\phi) \eta$. This means the trace does not separate into oscillator and lattice contributions. Thus, a restricted set of states appears, in which certain combinations of oscillators appear with certain lattice vectors. This set of states is generated by acting on the $|q=-1\rangle$ vacuum for the NS sector and on $\left|q=-\frac{1}{2}\right\rangle$ for the $\mathrm{R}$ sector [78].

We bypass this problem by the following trick. Introduce two auxiliary fermion fields, $\exp (\sigma)$ and $\exp (-\sigma)$. The boson, $\sigma$, has $\epsilon=1$ and $Q=2$. The $\beta-\gamma-\sigma$ system has supersymmetry in the sense that there is exact boson-fermion level degeneracy so that the partition function is unity. Actually, in the $\mathbf{R}$ sector, one must be careful to give zero modes such as $\gamma_{0}$ a small positive weight, $\delta$, and take $\delta$ to 0 after the calculation; otherwise $0 / 0$ arises. Wherever $\exp (\lambda \cdot \phi)(z)$ appears, replace it by $\exp (\lambda \cdot(\phi+\sigma))(z)$. Since operator-product expansions among the latter are all non-singular and since the system is supersymmetric, one might suspect that a correlation function of exponentials of $(\phi+\sigma)$ is one. We do not have a proof of this result, but we have checked it by saturating the trace by the first few levels. Furthermore, when the torus case is treated by the same method, one gets the $\phi$ correlation functions of refs. $[52,58,59]$, which were determined by computing the $\beta-\gamma$ path integral or by using complex function theory (methods (4) and (6) in the 
Introduction):

$$
\begin{gathered}
\left.\quad T \exp \left[\lambda_{n} \phi\left(z_{n}\right)\right] \ldots \exp \left[\lambda_{1} \phi\left(z_{1}\right)\right]\right\rangle_{\beta, \alpha}= \\
\left\{f(T) \prod_{m>\ell} \exp \left[\lambda_{m} \lambda_{\ell} g_{o s c}\left(z_{m}, z_{\ell}, T\right)\right]\right. \\
\left.\quad \times \Lambda\left(\beta, \alpha, 2, T,\left\{z_{k}\right\}\right) \prod_{m>\ell} \exp \left(\lambda_{m} \lambda_{\ell} \ln z_{m}\right)\right\}^{-1}
\end{gathered}
$$

Computing $\partial \phi$ terms is awkward, but we have derived a set of rules by taking limits of the exponential correlation function in Eq. (IV.18).

(1) Draw a line $\mathrm{q}$ for the $|q\rangle$ state. Take all possible contractions, including contractions to $\mathrm{q}$.

(2) If $\ell$ line are attached to possible ways. This leads to a factor of $p$ ! if $p$ points are involved and a fixed order is used.

(3) Associate the following factors with the contractions:

$$
\begin{aligned}
& \partial \underline{\underline{\phi}(z) e^{\lambda \phi}}(w) \rightarrow-\lambda\left[\frac{\partial}{\partial z} g_{o s c}(z, w, T)+\frac{1}{z}\right] \\
& \partial \phi(z) \partial \phi(w) \rightarrow \frac{-\partial}{\partial z} \frac{\partial}{\partial w} g_{o s c}(z, w, T) \\
& \partial \phi(z) \underset{q}{q} \rightarrow \frac{-\sum_{q} w(q) q}{\sum_{q} w(q)} \\
& \partial \phi\left(z_{m}\right) \ldots . \partial \phi\left(z_{2}\right) \partial \phi\left(z_{1}\right) \underbrace{q} \rightarrow \frac{-\sum_{q} w(q) \frac{q^{m}}{z_{m} \ldots z_{2} z_{1}}}{\sum_{q} w(q)}
\end{aligned}
$$

where

$$
w(q)=\exp \left[\frac{-1}{2} q(q+Q) T\right] \exp \left[i \pi q \alpha_{6}\right] \exp \left[q \sum_{i} \lambda_{i} \ln z_{i}\right]
$$

(4) The correlation function is the product of these contraction factors with the remaining exponential expectation, i.e., Eq. (IV.18). 
(5) Terms involving higher derivatives of $\phi$ are obtained by differentiating Eq. (IV.19) with respect to $z$ or $w$.

To clarify (2), consider the case in which $\partial \phi\left(z_{3}\right) \partial \phi\left(z_{2}\right) \partial \phi\left(z_{1}\right)$ is present. Then, there can be $0,1,2$, or 3 contractions with q points can be drawn. For two points in which $\partial \phi\left(z_{2}\right)$ and $\partial \phi\left(z_{1}\right)$ are contracted with the same point there are two diagrams:

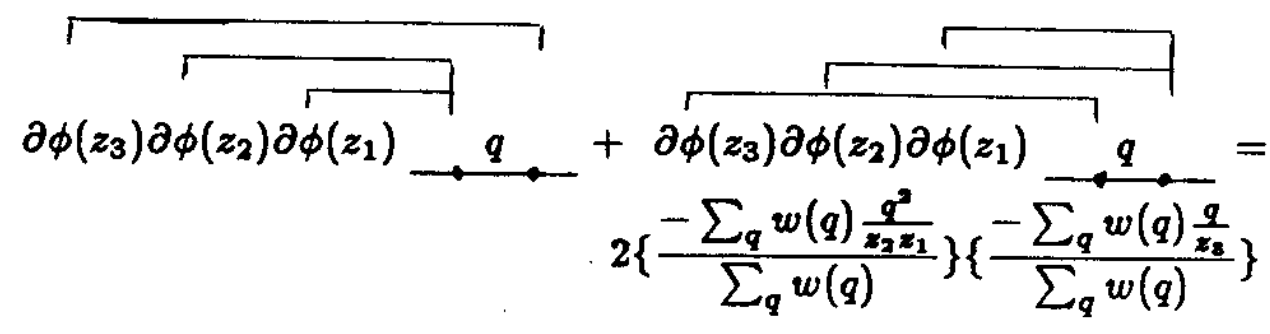

The above methods are easily adapted to any $\epsilon=-1$ system.

At this stage, we can compute any $\psi$ - $\phi$ correlation function. To implement the GSO projection, let

$$
\begin{aligned}
& \alpha_{i}=1-\alpha, i=1, \ldots, 5 \\
& \alpha_{6}=1+\alpha
\end{aligned}
$$

Then, the sectors (o), (v), (s) and (c) of the open superstring, defined in subsection III.E of [78], are weighted by $1,-(-1)^{\alpha},-1$ and $(-1)^{\alpha}$, respectively. Averaging over $\alpha$ selects sectors (o) and (s), i.e., the GSO-accepted NS sector and the GSO-accepted R sector with left-handed massless spinor.

Incorporating the cocycle factors and the above results, we find

$$
\begin{aligned}
& T\left\langle\exp \left(\lambda_{n} \cdot \phi\right)\left(z_{n}\right) \ldots . \exp \left(\lambda_{1} \cdot \phi\right)\left(z_{1}\right)\right\rangle_{\phi-\phi}= \\
& \sum_{\beta=0,+1 / 2} \frac{1}{2} \sum_{\alpha=0,1}\left\{[f(T)]^{-4} \prod_{m>\ell} \exp \left[\left\langle\lambda_{m} \cdot \lambda_{\ell}\right\rangle g_{o s c}\left(z_{m}, z_{\ell}, T\right)\right]\right\} \\
& \times\left\{\prod_{i=1}^{6}\left[\Lambda\left(\beta, \alpha_{i}, Q_{i}, T,\left\{z_{\ell}\right\}\right)\right]^{\epsilon_{i}} \prod_{m>\ell} \exp \left[\left\langle\lambda_{m} \cdot \lambda_{\ell}\right\rangle \ln z_{m}+i \pi \lambda_{m} M \lambda_{\ell}\right]\right\}
\end{aligned}
$$

where 


$$
\begin{aligned}
& Q_{i}=0, \quad \epsilon_{i}=1, i=1, \ldots, 5 \\
& Q_{6}=2, \quad \epsilon_{6}=-1 .
\end{aligned}
$$

In Eq. (IV.23) the one-loop contributions for Neveu-Schwarz and Ramond sectors are united into one lattice sum. Alternatively, for fixed $\beta$ and $\alpha$, Eq. (IV.23) can be expressed in terms of theta functions via Eqs. (IV.1) and (IV.16). The one-loop partition function for the $\psi$ - $\phi$ system is Eq. (IV.23) with all $\lambda_{l}=0$.

\section{Möbius Strip Amplitudes}

The Möbius strip differs from the annulus in that the two ends of Figure 1a are twisted before being joined. The twist operator $\exp \left(i \pi \tilde{L}_{0}\right)$, where $\tilde{L}_{0}$ is the evolution operator minus the $p^{2}$ term, sends $\sigma$ to $\pi-\sigma[27]$ when applied at time $\tau=0$. This operator effects the replacement $T \rightarrow T+i \pi$ in all correlation-function results [27]. The Möbius-strip rules are the same as the annulus ones. There is one $b_{0}$ insertion, and $T$ is integrated from 0 to $\infty$. The $z_{i}$ are integrated over appropriate ranges in the intervals from -1 to $-\exp (T)$ and 1 to $\exp (T)$. There is now no distinction between planar and non-planar amplitudes since there is only one boundary: the inner radius in Figure $1 \mathrm{~b}$ is first twisted and then is identified with the outer radius.

Summarizing, for the Möbius strip one substitutes $T \rightarrow T+i \pi$ in the correlation functions, but not in the rules.

\section{E. Boundary Operators and BRST Invariance}

Boundary operators for the annulus are easily found by the following method. For simplicity, consider the $X^{\mu}$ degrees of freedom. One would like to compute vertex operators with respect to the measure factor 


$$
\begin{aligned}
\int D X_{0}(\sigma) & \int_{X(\sigma, 0)=X(\sigma, T)=X_{0}(\sigma)} D X(\sigma, \tau) \exp [-S(X(\cdot, \cdot))] \\
= & \int Y(\sigma) \int D X(\sigma, \tau) \exp [-S(X(\cdot, \cdot))] \\
& \times \prod_{\sigma} \delta(X(\sigma, 0)-Y(\pi-\sigma, 0)) \delta(X(\sigma, T)-Y(\pi-\sigma, 0))
\end{aligned}
$$

The doubling of $\delta$-functions is reminiscent of the trick used in ref. [120].

Since $\delta(X(\sigma, T)-Y(\pi-\sigma, 0))$ is the twisted delta function, it is equivalent to the crosscap boundary operator in Eq. (II.2) except: (a) L and R oscillators and Fock-space states are associated with $\mathrm{X}$ and $\mathrm{Y}$ oscillators and states; (b) only $\mathrm{X}$ has been shifted in time, so $\exp (-n T)$ factors appear instead of $\exp (-2 n T)$ in Eq. (II.12); and (c) the zero modes of $\mathrm{X}$ and $\mathrm{Y}$ are different, resulting in a factor of $\exp \left(-\alpha^{\prime} T p_{L}^{2}\right)$ in Eq. (II.2) where $p_{L}^{\mu}$ is the center-of-mass momentum of $X^{\mu}$. Similarly, $\delta(X(\sigma, 0)-Y(\pi-\sigma, 0))$ can be represented by a right-bracket operator, $|C, 0\rangle$, which is the hermitian conjugate of $\langle C, 0|$. See, for example, ref. [107] for the hermitian-conjugation rules. The doubling trick introduces two sets of variables. In what follows, we distinguish these by superscripts (1) and (2).

The annulus amplitudes can be represented as

$$
\int d T\left\langle C, \frac{T}{2}\left|b_{0}^{(1) L} V_{n} \ldots V_{1}\right| C, 0\right\rangle
$$

In the superstring case, however, one cannot directly use Eqs. (II.4) because this would sum over the entire ghost-extended lattice. The comments in the paragraph below Eq. (IV.17) are applicable here.

It is straightforward to show that Eq. (IV.26) is equivalent to taking the trace in a coherent-state basis. For the oscillators, rewrite 


$$
\begin{aligned}
& \exp \left(\frac{-\alpha_{-n}^{X} \alpha_{-n}^{Y}(-1)^{n}}{n}\right)=\frac{1}{\pi} \int d^{2} \lambda \exp \left[-\lambda^{*} \lambda+\frac{\lambda}{\sqrt{n}} \alpha_{-n}^{X}-\frac{(-1)^{n} \lambda^{*}}{\sqrt{n}} \alpha_{-n}^{Y}\right] \\
& \exp \left(\frac{-\alpha_{n}^{X} \alpha_{n}^{Y}}{n}(-1)^{n} e^{-n T}\right)=\frac{1}{\pi} \int d^{2} \tilde{\lambda} \exp \left[-\tilde{\lambda}^{*} \tilde{\lambda}+\frac{e^{-n T}}{\sqrt{n}} \tilde{\lambda}^{*} \alpha_{n}^{X}-\frac{(-1)^{n} \tilde{\lambda} \alpha_{n}^{Y}}{\sqrt{n}}\right]
\end{aligned}
$$

The trace over $\mathrm{Y}$ oscillators is then possible, since the vertex operators in Eq. (IV.26) do not depend on $Y(\sigma, 0)$. Next, do the integrals over $\tilde{\lambda}$ and $\tilde{\lambda}^{*}$. In a few lines of algebra the standard coherent-state trace representation [27] emerges.

The BRST decoupling of spurious states follows from

$$
\begin{aligned}
& \int d T\left\langle C, \frac{T}{2}\left|b_{0}^{(1) L} V_{n} \ldots V_{1}\left\{Q, V_{0}\right\}\right| C, 0\right\rangle \\
& =\int d T\left\langle C, \frac{T}{2}\left|L_{0}^{(1)} V_{n} \ldots V_{1} V_{0}\right| C, 0\right\rangle \\
& =\int d T \frac{d}{d T}\left\langle C, \frac{T}{2}\left|V_{n} \ldots V_{1} V_{0}\right| C, 0\right\rangle=\text { boundary terms. }
\end{aligned}
$$

To derive Eq. (IV.28), we have used that $Q_{B R S T}=Q_{B R S T}^{(1)}+Q_{B R S T}^{(2)}$ annihilates $\langle C, T / 2|$ and $|C, 0\rangle$. Also, $\left[Q_{B R S T}, V_{j}\right]$ is zero since physical vertex operators $V_{j}$ satisfy $\left[Q_{B R S T}^{(1)}, V_{j}\right]$ $=0$ and are independent of $Y(\sigma, 0)$ and (2) variables. Whether spurious states decouple depends on whether the boundary terms in Eq. (IV.28) vanish. The discussion here is analogous to that at the end of Section II.

For the Möbius strip, $\langle C, T / 2|$ is replaced by $\left\langle C, \frac{1}{2} T+i \pi\right|$ while $|C, 0\rangle$ remains unchanged, i.e., both a twisted and an untwisted delta function are used. The argument for the BRST decoupling of spurious states for the Möbius strip is similar to that in Eq. (IV.28) 
A. Introduction

One-loop closed-string corrections involve the torus and Klein-bottle topologies. The torus is obtained from a cylinder of length $T$ by twisting one end by an angle $\theta$ and then joining the ends. Correspondingly, take the vertical range in Figure 1a to run from $-\pi$ to $\pi$ instead of 0 to $\pi$. The upper and lower edges are identified naturally when the variable $z=\exp (\tau+i \sigma)$ is used. The remaining identification of opposite ends must be done by hand. In the $\mathrm{z}$ plane, the configuration is an annulus with inner radius 1 and outer radius $\exp (T)$ and the two circular boundaries identified.

The correlation functions on the torus have been computed in refs. $[50,51,52,55,58$, 59], for example, by methods (4) and (6) of the Introduction. This suffices to determine amplitudes. In view of this previous work, we will be brief. With a few changes, the results of Section IV directly yield the torus and Klein-bottle amplitudes.

One-loop amplitudes for tachyons and massless states have been previously obtained and are given in ref. [27] in light-cone gauge. Results in this Section differ from [27] in that general amplitudes are obtained and a covariant approach is used. Again, conformalfield-theoretic methods facilitate the computations.

B. Rules

(1) The amplitude is

$$
\int d T d \theta\left\langle b_{0}^{L} b_{0}^{R} V_{n} \ldots V_{1}\right\rangle
$$

where $n-1$ integral-type vertices and one $c^{L} c^{R}$-type vertex are used. In the case of the superstring, the left and right $\phi$-ghost charges of the vertices must sum to 0 . The vertex operators are those used at tree level. 
(2) Let

$$
\tau=\frac{i}{2 \pi}(T+i \theta)
$$

be the complex modular parameter. The integration region for $\tau$ is $F: F=\left\{\tau \mid-\frac{1}{2}<R e \tau\right.$ $\left.<\frac{1}{2}, I m \tau>0,|\tau|>1\right\}$. For $z_{i}$ associated with the $i^{\text {th }}$ integral vertex, the integration domain is the annulus: $1<\left|z_{i}\right|<\exp (T)$.

(3) The torus correlation functions are related to the ones in Section IV as explained below in Section V.C.

Twisting by and integrating over $\theta$ implements the $L_{0}^{L}-L_{0}^{R}=0$ constraint in the trace. The equivalence of tori with modular parameter $\tau$ and $-\frac{1}{r}$ further restricts the $\tau$-integration region to $F[16]$. Note that BRST invariance requires the measure $d^{2} \tau$ to be flat in rule (1). The familar $d^{2} \tau(I m \tau)^{-2}$ measure is generated by the correlation function, as explained in Section VI.

C. Correlation Functions

The evolution operator for the torus is $\exp \left[-(T+i \theta) L_{0}^{L}-(T-i \theta) L_{0}^{R}\right]$. Since closedstring vertex operators are products of left- and right-moving vertex operators, an amplitude factorizes into the product of a left-moving piece and a right-moving piece. This system corresponds to the annulus with $T \rightarrow T+i \theta$ for the left sector and $T \rightarrow T-i \theta$ for the right sector. With these substitutions, the results for correlation functions in Section IV.C are applicable here.

A complication arises for $X_{\text {closed }}(\sigma, \tau)$ because left and right decompositions have the same center-of-mass coordinates (see Eq. (I.4)). The closed-string bosonic propagator is 


$$
\begin{aligned}
T, \theta\left\langle X_{\text {closed }}\left(\sigma^{\prime}, \tau^{\prime}\right) X_{\text {closed }}(\sigma, \tau)\right\rangle & =\frac{\alpha^{\prime}}{2}\left[g_{o s c}\left(z^{\prime}, z, T+i \theta\right)+g_{\text {osc }}\left(\bar{z}^{\prime}, \bar{z}, T-i \theta\right)\right. \\
& \left.+2 g_{\text {zero }}\left(z^{\prime}, z, T\right)\right] \\
& =\frac{\alpha^{\prime}}{2} g_{\text {closed }}\left(z^{\prime}, \bar{z}^{\prime}, z, \bar{z}, T, \theta\right)
\end{aligned}
$$

so that

$$
\begin{aligned}
& T, \theta\left\langle\exp \left[k_{n} \cdot X\left(z_{n}, \bar{z}_{n}\right)\right] \ldots \exp \left[k_{1} \cdot X\left(z_{1}, \bar{z}_{1}\right)\right]\right\rangle_{X} \\
& \quad=T, \theta\langle 1\rangle_{X} \prod_{j>i} \exp \left[\frac{\alpha^{\prime}}{2} k_{j} \cdot k_{i} g_{\text {closed }}\left(z_{j}, \bar{z}_{j}, z_{i}, \bar{z}_{i}, T, \theta\right)\right]
\end{aligned}
$$

where

$$
T, \theta\langle 1\rangle_{X}=\{f(T+i \theta) f(T-i \theta)\}^{-D}\left(2 \pi \alpha^{\prime} T\right)^{\frac{-D}{2}}
$$

Eq. (V.3) is similar to Eq. (IV.7) if $2 \alpha^{\prime} \rightarrow \alpha^{\prime} / 2$.

Derivative terms for $\partial X$ are evaluated in a manner similar to Eq. (IV.10) with $g_{X}(z, w, T) \rightarrow g_{c l o s e d}(z, \bar{z}, w, \bar{w}, T, \theta)$.

As an example of how annulus results carry over to torus ones, the b-c correlation function is

$$
T, \theta\left\langle b_{0}^{R} b_{0}^{L} c^{L}(z) c^{R}(\bar{z})\right\rangle_{b c}=\left|T_{+i \theta}\left\langle b_{0} c(z)\right\rangle_{b c}\right|^{2}=z \bar{z} e^{2 T}[f(T+i \theta) f(T-i \theta)]^{2}
$$

where $\left\langle b_{0} c(z)\right\rangle$ is given in Eq. (IV.11).

Correlation functions for bosons associated with a bosonization procedure are obtained by factoring them into a left-moving piece times a right-moving piece. For the left sector, the correlation functions are Eqs. (IV.13-IV.16) with $T \rightarrow T+i \theta$ and with L subscripts appearing on the variables $\lambda_{\ell}, \beta, \alpha_{i}, Q_{i}$ and the matrix M. For the right sector, $T \rightarrow T-i \theta$, $z_{i} \rightarrow \bar{z}_{i}$, and $R$ subscripts are attached instead. 


\section{BRST Invariance}

Boundary operators for the torus are constructed by the same doubling technique used for the annulus in Section IV. Twice as many right oscillators and twice as many left oscillators are introduced. Since left- and right-moving sectors decouple, torus $\langle C, T\rangle=$ ${ }_{R}\left\langle C,\left.T\right|_{L}\langle C, T| \text { and } \mid C, 0\right\rangle_{\text {torue }}=|C, 0\rangle_{L}|C, 0\rangle_{R}$

Spurious states, associated with BRST anticommutators, lead to $\frac{d}{d T}$ and $\frac{d}{d \theta}$ derivatives of the integrand. This follows from $b_{0}^{L} b_{0}^{R}=-\left(b_{0}^{L}+b_{0}^{R}\right)\left(b_{0}^{L}-b_{0}^{R}\right) / 2,\left\{Q_{B R S T}, b_{0}^{L}+b_{0}^{R}\right\}=$ $L_{0}^{L}+L_{0}^{R}$, and $\left\{Q_{B R S T}, b_{0}^{L}-b_{0}^{R}\right\}=L_{0}^{L}-L_{0}^{R}$. The first anticommutator can be replaced by $\frac{d}{d T}$ and the second by $\frac{d}{d \theta}$. The effect of $\frac{d}{d \theta}$ disappears if an amplitude is periodic in $\theta$ with period $2 \pi$. The question of decoupling of spurious states then rests on whether the $I m \tau$-boundary terms vanish at infinity.

\section{E. The Klein Bottle}

The Klein bottle is obtained from a cylinder of length $T$ by identifying the $\sigma$ variable at one end with the combination $2 \pi-\sigma+\theta$ at the other. Converting $\sigma$ to $2 \pi-\sigma$ interchanges $z$ and $\bar{z}$ in mode expansions and, therefore, R and L oscillators. As a consequence, Kleinbottle amplitudes can be related to annulus-like ones, where the width of the annulus is $2 \pi$ and the length is $2 T$, as explained in ref. [27]. This is based on

$$
\begin{aligned}
\sum_{\psi_{R} \psi_{L}}\left\{{ }_{R}\left\langle\left.\psi_{R}\right|_{L}\left\langle\psi_{L}|\ldots| \psi_{L}\right\rangle_{L} \mid \psi_{R}\right\rangle_{R}\right\}_{\text {Klein bottle }} & =\sum_{\psi_{R} \psi_{L}}{ }_{R}\left\langle\left.\psi_{R}\right|_{L}\left\langle\psi_{L}|\ldots| \psi_{R}\right\rangle_{L} \mid \psi_{L}\right\rangle_{R} \\
& =\sum_{\psi_{R} \psi_{L}}{ }_{L}\left\langle\psi_{L}|\ldots| \psi_{R}\right\rangle_{L} \quad{ }_{R}\left\langle\psi_{R}|\ldots| \psi_{L}\right\rangle_{R} \\
& =\sum_{\psi_{L}}\left\langle\psi_{L}|\ldots \ldots| \psi_{L}\right\rangle
\end{aligned}
$$

More precisely,

$$
\begin{aligned}
& \left\langle O_{n}^{L}\left(z_{n}\right) O_{n}^{R}\left(\bar{z}_{n}\right) \ldots O_{1}^{L}\left(z_{1}\right) O_{1}^{R}\left(\bar{z}_{1}\right)\right\rangle_{K B} \\
= & \pm\left.\left\langle O_{n}^{R}\left(y_{n}\right) \ldots O_{1}^{R}\left(y_{1}\right) O_{n}^{L}\left(z_{n}\right) \ldots O_{1}^{L}\left(z_{1}\right)\right\rangle\right|_{y_{i}=\bar{z}_{i} e^{T+i \theta}} \exp \left[H^{R}(T+i \theta)\right]
\end{aligned}
$$


where a minus sign can arise from reordering fermionic operators and $H^{R}=h_{1}^{R}+\ldots+h_{n}^{R}$ is the sum of the right conformal weights of $O_{1}^{R}, \ldots, O_{n}^{R}$. The Klein-bottle amplitude appears only for unoriented strings. Such strings must have a left-right symmetry between operators. Thus, it makes sense to replace right operators by left operators in Eq. (V.6). Effectively, the evolution operator $\exp \left(-(T+i \theta) H_{L}\right)$ is used to evolve the system from $\tau=0$ to $\tau=T$, while $\exp \left(-(T-i \theta) H_{L}\right)$ is used from $\tau=T$ to $\tau=2 T$. A Klein-bottle correlation function is therefore equivalent to one in which all right-handed operators are replaced by their left-handed counterparts shifted in $\tau$ by $T$ and in $\sigma$ by $\theta$. A factor of $\exp \left[h^{R}(T+i \theta)\right]$ arises because $O^{R}(\bar{z})=\sum_{n} O_{n}^{R} \bar{z}^{-n-h^{R}}$ and the modes $O_{n}^{R} \rightarrow O_{n}^{R} \exp [-n(T+i \theta)]$ are shifted forward in time.

The torus rules apply to the Klein-bottle case with the following exception. In rule (2), the integration region is 0 to $\infty$ for $T$ and $-\pi$ to $\pi$ for $\theta$. However, for $z_{i}$ associated with the $i^{\text {th }}$ integral vertex, the integration domain is still $1<\left|z_{i}\right|<\exp (T)$. The difference in the modular integration range arises because $\tau \rightarrow-\frac{1}{r}$ does not generate an equivalent Klein bottle.

Eq. (V.6) is valid for $\psi$ operators with ghost charge, written in the form of Eq. (I.1). In removing derivative terms, one must first make the replacement of left operators by right operators, i.e., $\bar{\partial} \phi^{R}(\bar{z}) \rightarrow \partial_{y} \phi^{L}(y)$, and then use Wick's theorem. Finally, the argument is evaluated at $\bar{z} \exp (T+i \theta)$, i.e., $y \rightarrow \bar{z} \exp (T+i \theta)$.

The revelant b-c correlation function is

$$
T, \theta\left\langle b_{0}^{R} b_{0}^{L} c^{L}\left(z_{n}\right) c^{R}\left(\bar{z}_{n}\right)\right\rangle_{K B}=z_{n} \bar{z}_{n} e^{2 T}[f(2 T)]^{2}
$$

Correlation functions for the X-fields are more difficult because Eq. (V.6) applies only to the oscillators, not to the center-of-mass coordinates. The zero-mode contribution is the same as in the torus case. For example, 


$$
\begin{aligned}
T, \theta & \left\langle\exp \left[k_{n} \cdot X\left(z_{n}, \bar{z}_{n}\right)\right] \ldots \exp \left[k_{1} \cdot X\left(z_{1}, \bar{z}_{1}\right)\right]\right\rangle_{K B} \\
= & \left\{\left(\frac{1}{2 \pi \alpha^{\prime} T}\right)^{\frac{D}{2}} \prod_{j>i}\left[z_{j} \bar{z}_{j} e^{-\frac{1}{R \cdot T}\left(\ln \mid \frac{s_{j}}{s_{i}}\right)^{2}}\right]^{\frac{\alpha^{\prime}}{2} k_{j} \cdot k_{i}}\right\} \\
& \times\left\{[ f ( 2 T ) ] ^ { - D } \prod _ { j > i } \operatorname { e x p } \left[\frac{\alpha^{\prime}}{2} k_{j} \cdot k_{i}\left(g_{o s c}\left(z_{j}, z_{i}, 2 T\right)+g_{o s c}\left(\bar{z}_{j}, \bar{z}_{i}, 2 T\right)\right)\right.\right. \\
& \left.\times \prod_{j, i} \exp \left\{\frac{\alpha^{\prime}}{2} k_{j} \cdot k_{i} g_{o s c}\left(z_{j}, \bar{z}_{i} e^{T+i \theta}, 2 T\right)\right]\right\}
\end{aligned}
$$

where $g_{\text {oac }}$ is given in Eq. (IV.1). The first and second terms in curly brackets in Eq. (V.8) are, respectively, the zero-mode and oscillator contributions.

The Wick factors associated with derivative terms are

$$
\begin{aligned}
& \partial X^{\mu}(z) e^{k \cdot X}(w, \tilde{w}) \rightarrow \alpha^{\prime} k^{\mu} \partial_{z}\left\{g_{z e r o}(z, \bar{z}, w, \bar{w}, T)+\frac{1}{2} g_{o s c}(z, w, 2 T)\right. \\
& \left.+\frac{1}{2} g_{o s c}\left(\bar{w} e^{T+i \theta}, z, 2 T\right)\right\} \\
& \bar{\partial} X^{\mu}(\bar{z}) e^{k \cdot X}(w, \bar{w}) \rightarrow \alpha^{\prime} k^{\mu}\left\{\bar{\partial}_{\bar{z}} g_{z e r o}(z, \bar{z}, w, \bar{w}, T)\right. \\
& \left.+\frac{1}{2} \bar{\partial}_{\bar{z}} g_{o s c}(\bar{z}, \bar{w}, 2 T)+\left.\frac{1}{2} \partial_{y} g_{o s c}(y, w, 2 T)\right|_{y=\bar{z} e^{T+i o}}\right\} \\
& \partial X^{\mu}(z) \partial X^{\nu}(w) \rightarrow \eta^{\mu \nu} \alpha^{\prime} \partial_{z} \partial_{w}\left\{g_{z e r o}(z, \bar{z}, w, \bar{w}, T)+\frac{1}{2} g_{o o c}(z, w, 2 T)\right\} \\
& \underset{\bar{\partial} X^{\mu}(\bar{z}) \bar{\partial} X^{\nu}}{\longrightarrow}(\bar{w}) \rightarrow \eta^{\mu \nu} \alpha^{\prime} \bar{\partial}_{\bar{z}} \bar{\partial}_{\bar{w}}\left\{g_{z e r o}(z, \bar{z}, w, \bar{w}, T)+\frac{1}{2} g_{o s c}(\bar{z}, \bar{w}, 2 T)\right\} \\
& \bar{\partial} X^{\mu}(\bar{z}) \partial X^{\nu}(w) \rightarrow \eta^{\mu \nu} \alpha^{\prime} \partial_{w}\left\{\bar{\partial}_{\bar{z}} g_{z e r o}(z, \bar{z}, w, \bar{w}, T)+\left.\frac{1}{2} \partial_{y} g_{o s c}(y, w, 2 T)\right|_{y=\bar{z} e^{T+i \bullet}}\right\}
\end{aligned}
$$

where again a separation of zero-mode and oscillator terms is necessary.

With the above techniques, any Klein-bottle amplitude can be computed. 


\section{MODULAR INVARIANCE}

The purpose of this Section is to present a modified approach in which modular invariance of arbitrary torus amplitudes is manifest.

For one-loop closed-string amplitudes, invariance under the modular group

$$
\left(\nu_{i}, \tau\right) \rightarrow\left(\frac{\nu_{i}}{c \tau+d}, \frac{a \tau+b}{c \tau+d}\right), \quad\left(\begin{array}{ll}
a & b \\
c & d
\end{array}\right) \epsilon S L(2, Z) .
$$

has been a guiding principle in constructing consistent string theories. In the modern BRST approach it is not obvious that the amplitudes in Section $V$ are modular invariant.

Frequently, the question of modular invariance is addressed only for the partition function, tachyonic amplitudes, or massless amplitudes. Let us demonstrate that the rules in Section V give modular-invariant amplitudes for arbitrary states. This is possible by rearrangment of the measure and pieces of the correlation functions. The resulting expression is the most convenient for the torus topology. Below we discuss a purely conformal field-theoretic approach that directly yields this manifestly modular-invariant formulation. The extension to other topologies is briefly sketched. This description offers a treatment of higher-loop amplitudes.

To show modular invariance it is convenient to change variables from $\mathrm{z}$ and $T+i \theta$ to

$$
\begin{gathered}
\nu=\frac{1}{(2 \pi i)} \ln z \\
\tau=\frac{i}{(2 \pi)}(T+i \theta) .
\end{gathered}
$$

Since Eq. (VI.2a) is a conformal transformation, any amplitude must be form-invariant by construction. The effect on primary fields is given by their conformal weights:

$$
O(z, \bar{z})=(2 \pi i z)^{h_{L}}(-2 \pi i \bar{z})^{h_{R}} O(\nu, \bar{\nu}) .
$$


For the propagators in Eqs. (IV.1) and (V.2) this means

$$
\begin{aligned}
g_{\text {osc }}\left(z, z^{\prime}\right)+\ln z & =G_{L}\left(\nu-\nu^{\prime} \mid \tau\right)+\ln \left(2 \pi i \sqrt{z z^{\prime}}\right) \\
g_{\text {closed }}\left(z, z^{\prime}, \bar{z}, \bar{z}^{\prime}\right) & =G\left(\nu-\nu^{\prime} \mid \tau\right)+\ln \left(4 \pi^{2}\left|z z^{\prime}\right|\right)
\end{aligned}
$$

where

$$
\begin{aligned}
G_{L}(\nu \mid \tau) & =\ln \left\{\Theta\left[\begin{array}{l}
\frac{1}{2} \\
\frac{1}{2}
\end{array}\right](\nu \mid \tau) / \Theta^{\prime}\left[\begin{array}{l}
\frac{1}{2} \\
\frac{1}{2}
\end{array}\right](0 \mid \tau)\right\} \\
G(\nu \mid \tau) & =G_{L}(\nu \mid \tau)+G_{L}(\nu \mid \tau)^{*}-2 \pi(I m \nu)^{2} / I m \tau
\end{aligned}
$$

are the scalar propagators for chiral bosons $\phi(\nu)$ and real fields $X(\nu, \bar{\nu})$, respectively.

Conformal invariance guarantees the cancellation of the individually appearing $z$ and $\bar{z}$ factors from Eqs. (VI.3) and (VI.4). However, the propagators in Eq. (VI.4) are independent of background charge $Q$. One therefore has to compensate for the $Q$-dependent part of $\left(h_{L}, h_{R}\right)$ in Eq. (VI.3). The rules of Sections IV and V can then be carried over completely under the replacements

$$
\begin{aligned}
(z, \bar{z}) & \rightarrow(\nu, \bar{\nu}) \\
g_{\text {osc }}\left(z, z^{\prime}\right)+\ln z & \rightarrow G_{L}\left(\nu-\nu^{\prime}\right) \\
g_{\text {closed }}\left(z, z^{\prime}, \bar{z}, \bar{z}^{\prime}\right) & \rightarrow G\left(\nu-\nu^{\prime}\right) \\
\exp (q \phi)(z) & \rightarrow(2 \pi i z)^{q Q / 2} \exp (q \phi)(\nu) .
\end{aligned}
$$

In terms of $\nu$ and $\tau$, Eqs. (V.4) and (V.5) become

$$
\begin{aligned}
r\langle 1\rangle_{X} & =\left(4 \pi^{2} \alpha^{\prime} I m \tau\right)^{-D / 2}|\eta(\tau)|^{-2 D} e^{-(D / 6) \pi I m r} \\
r\left\langle b_{0}^{L} b_{0}^{R} c^{L}(\nu) c^{R}(\bar{\nu})\right\rangle_{b c} & =\left(1 / 4 \pi^{2}\right)|\eta(\tau)|^{4} e^{(13 / 3) \pi I m \tau} .
\end{aligned}
$$

Here, $\eta(\tau)=f(-2 \pi i \tau) e^{\pi i r / 12}$ denotes the Dedekind eta function. Assembling Eqs. (IV.1316) and (IV.18) we obtain for the superstring:

$$
{ }_{r}\langle 1\rangle_{[\alpha, \beta]}=(-)^{2(\alpha+\beta)} \eta(\tau)^{-4}\left\{\Theta\left[\begin{array}{l}
\alpha \\
\beta
\end{array}\right](0 \mid \tau)\right\}^{4} e^{(4 / 3) \pi i \tau}
$$


where $[\alpha, \beta]$ denotes the spin structures with $\alpha$ and $\beta$ equal to 0 or $\frac{1}{2}$. Note that the exponentials in Eq. (VI.7) cancel for D = 26, whereas in the product $E q .(V I .7) \times|E q .(V I .8)|^{2}$ they cancel for $D=10$. This occurs because the tachyon masses were originally chosen in the critical dimensions.

Let us now investigate the behaviour of measure and correlation functions under modular transformations, Eq. (VI.1). In terms of the rules in Sections IV and V, we must show that the correlation functions as evaluated in terms of propagators and lattice sums do indeed mimic the transformation properties of the operator $O$. Also, the remaining measure factors, e.g., Eq. (VI.7), must be shown to combine with the flat measure $d^{2} \tau$ to give modular-invariant combinations. From the literature [113], we have

$$
\begin{aligned}
G & \rightarrow G-\ln |c \tau+d|^{2} \\
\partial G & \rightarrow(c \tau+d) \partial G \\
\bar{\partial} G & \rightarrow(c \bar{\tau}+d) \bar{\partial} G \\
G^{L} & \rightarrow G^{L}-\ln (c \tau+d)+i \pi c \nu^{2} /(c \tau+d) \\
\Theta\left[\begin{array}{l}
\alpha \\
\beta
\end{array}\right](\nu) & \rightarrow \epsilon(c \tau+d)^{1 / 2} \exp \left(i \pi c \nu^{2} /(c \tau+d)\right) \Theta\left[\begin{array}{l}
\alpha^{\prime} \\
\beta^{\prime}
\end{array}\right](\nu) \\
\eta & \rightarrow \epsilon^{\prime}(c \tau+d)^{1 / 2} \eta \\
I m \tau & \rightarrow I m \tau /|c \tau+d|^{2}
\end{aligned}
$$

where $\epsilon, \epsilon^{\prime}$ are phases. Invariant combinations are

$$
(I m \tau)^{1 / 2}|\eta(\tau)|^{2}, \quad d^{2} \tau /(I m \tau)^{2}, \quad \sum_{\{\alpha, \beta]} \epsilon[\alpha, \beta, N]\left\{\Theta\left[\begin{array}{l}
\alpha \\
\beta
\end{array}\right](0 \mid \tau) /|\eta(\tau)|\right\}^{N}
$$

Using

$$
\sum_{i<j} k_{i} \cdot k_{j}=-(1 / 2) \sum_{i} k_{i}^{2}
$$

for $\sum_{i} k_{i}=0$ and the analogue for $\lambda_{i}$, it is easy to see that the modular weights of the Wick contractions correspond to the conformal dimensions of the original operators for 
vanishing background charges. This is also true for the rules for derivatives of $\mathrm{X}$ and $\phi$, Eqs. (IV.10) and (IV.17). For the full amplitude, the effect of nonzero $Q$ can be ignored because linear terms in $h_{i}$ sum to zero due to the vanishing total background charge. The lattice sum does not lead to additional conformal weights but displays some anomalous $\nu$ dependent behavior under Eq. (VI.9). However, the $\nu$-dependence in the transformations of $G^{L}$ and $\Theta$ in Eq. (VI.9) are seen to cancel each other in Eqs. (IV.13-16) after $\sum_{i} k_{i}=0$ is used. One may also verify that the phases $\epsilon[\alpha, \beta, N]$ in Eq. (VI.10) coincide with the ones in Eqs. (IV.16) or (VI.8) obtained by imposing the GSO projection, so that the sum over spin structures creates a modular invariant. Summarizing, any correlation function is a modular function in $\tau$ and $\tilde{\tau}$ of weights equal to the left and right total conformal dimensions, respectively, after the appropriate sum over spin structures. Hence, the zintegrated correlation $\left\langle b_{0}^{L} b_{0}^{R} V \int \ldots V \int_{V^{c}}\right\rangle$ gives rise to a factor $|c \tau+d|^{4}$ due to the b-ghost insertions, as can also be seen from Eq. (VI.7). This factor cancels with the transformation of the flat measure $d^{2} r$.

The modular measure and the correlation functions may now be written as independent invariants by rearrangment, recalling $V^{c}=c^{L} c^{R} V^{0}$ :

$$
\begin{aligned}
d^{2} \tau & \rightarrow d^{2} \tau /(I m \tau)^{2} \\
r\left\langle b_{0}^{L} b_{0}^{R} c^{L}(\nu) c^{R}(\bar{\nu})\right\rangle_{b c} & \rightarrow\left(1 / 2 \pi^{2}\right) I m \tau|\eta(\tau)|^{4} \\
{ }_{r}\left\langle V \int_{\ldots V} \ldots V^{0}\right\rangle_{X, \phi} & \rightarrow{ }_{r}\left\langle V \int^{\int . . V} \int_{\left.I m \tau V^{0}\right\rangle_{X, \phi} .}\right.
\end{aligned}
$$

This concludes the proof, which applies to the bosonic string as well as to superstrings. The same techniques should allow its extension to heterotic strings with modular-invariant partition function. The invariant modular integration measure is provided just by the bghost insertions. As expected from the locality of vertex operators, modular invariance of the amplitudes is a direct consequence of conformal invariance.

The manifestly modular-invariant formulation of orientable closed-string one-loop amplitudes can be obtained directly from the Polyakov path integral. Let us consider the 
ordinary $D=10$ superstring. If bosonization is used [73], in the superconformal gauge one has to perform quadratic field integrations in ten coordinates $\mathrm{X}$, four ghosts $b, \bar{b}, c, \bar{c}$, and seven compact bosonic degrees of freedom $\phi^{m}(m=1, \ldots, 6)$ and $\chi$. All these fields have arguments on a torus, conveniently parametrized by the "flat" variable $\nu \in C /(Z+\tau Z)$.

Due to the single Killing vector on the torus, one c-ghost zero mode has to be saturated in the path integral. This is accomplished by choosing one vertex of the c-type. The modulus $\tau$ corresponds to a metric degree of freedom that cannot be gauged away. Its integration yields an insertion of

$$
\int\left(d^{2} \nu / I m \tau\right) b^{L}(\nu, \bar{\nu}) \int\left(d^{2} \nu^{\prime} / I m \tau\right) b^{R}\left(\nu^{\prime}, \bar{\nu}^{\prime}\right)
$$

The $\mathrm{X}$ and $b, c$ integrals can be solved by standard methods. The quadratic piece in the exponent and the $b, c$ insertions yield determinants of the kinetic operators $(\partial \bar{\partial})_{0}$ and $\bar{\partial}_{1}$, zero modes excluded, on a parallelogram with periodic boundary conditions representing the cut torus. There are various ways to regulate these determinants, of which zeta-function regularization is favoured for its scale invariance. This yields the modular-covariant parts of Eq. (VI.7) for any $D$; see, for example, refs. [34,36,37,48,51,55]. Vertex operators lead to linear terms in the exponent and to insertions of derivatives of $\mathrm{X}$. These are treated by Wick's theorem, yielding the familiar Koba-Nielsen term and derivatives thereof. The propagator in question is G, given in Eq. (VI.5). It generates pieces that do not factorize holomorphically.

The situation is slightly more involved for the degrees of freedom $\phi^{m}$ because these take values on the maximal torus of $D_{5,1}[81]$. This allows for classical soliton configurations with nonzero winding numbers $\left(n^{m}, m^{m}\right)$ in the bosonization lattice with respect to the two homology cycles [51]. Along the lines of method (4) of the Introduction, we can split $\phi=\phi_{n, m}+\phi_{\text {quantum }}$. The quantum fluctuations are like those of the $\mathrm{X}$ fields because background charges do not couple on the torus. The lattice sum over the distinct soliton sectors may be performed explicitly. The result is a modulus-squared times a nonanalytic 
piece cancelling that from the Koba-Nielsen term. This allows holomorphic factorization in the compact degrees of freedom, which in turn makes the method applicable to heterotic strings as well. Effectively, left-moving quantum contributions obtain from the chiral determinants

$$
\left[\operatorname{det}^{\prime} \bar{\partial}_{0}\right]^{4}=(I m \tau)^{-1} \eta(\tau)^{-4}
$$

and from Wick's method using the propagator $G^{L}$ (Eq.(VI.5)). For the left-moving part of the lattice sum we find in the notation of Eq. (IV.13):

$$
\begin{aligned}
\{\text { lattice factor }\}_{[\alpha, \beta]}= & I m \tau(-)^{2(\alpha+\beta)} \prod_{m}\left\{\Theta\left[\begin{array}{l}
\alpha \\
\beta
\end{array}\right]\left(\sum_{i} \lambda_{i}^{m} \nu_{i}\right)\right\}^{\epsilon_{m}} \\
& \times \prod_{i<j} \exp \left\{i \pi\left(\lambda_{i} \cdot M \lambda_{J}\right)\right\} \delta\left(\sum_{i} \lambda_{i}\right)
\end{aligned}
$$

where $\epsilon_{m}=(1,1,1,1,1,-1)$ and $[\alpha, \beta]$ denotes the spin structure. The product of Eqs. (VI.14), (VI.15) and the Koba-Nielsen term coincides with the result from Eqs. (IV.13-16) and (IV.18) after the change of variables. Note that the sum over the GSO-restricted odd self-dual lattice $\Gamma_{5,1}[78]$ generates the modular-invariant sum over spin structures. Derivatives of $\phi^{m}$ may be included in Eq. (VI.15) by differentiating with respect to some $z_{j}$. There is one subtlety in the argument leading to Eq. (VI.15): the lattice sum for $\phi^{6}$ diverges due to its timelike metric. However, Atick and Sen [58] and Verlinde and Verlinde [52] have shown by different methods that the contribution of a timelike boson is just the inverse of an ordinary spacelike one.

Finally, the $\chi$ system gives rise to a factor that cancels the determinants from $\phi^{5}$ and $\phi^{6}$. Actually, this is already included in Eq. (VI.14).

The method outlined above may be applied to any string theory including toroidal compactifications.

Other one-loop topologies can be derived from the torus by the method of images [45-47]. The annulus, the Möbius strip and the Klein bottle may be represented as the 
torus modulo particular involutions $\nu \rightarrow f(\nu)$. Wick's rules for the torus can then be taken over after the replacement

$$
G\left(\nu, \nu^{\prime}\right) \rightarrow \frac{1}{2}\left\{G\left(\nu, \nu^{\prime}\right)+G\left(f(\nu), \nu^{\prime}\right)+G\left(\nu, f\left(\nu^{\prime}\right)\right)+G\left(f(\nu), f\left(\nu^{\prime}\right)\right)\right\}
$$

and a similar replacement for $G^{L}$. Factors from determinants are modified to match the changed boundary conditions. One must take the lattice sum only over instanton configurations that are even under $f$. This reproduces the results of Sections IV and V, where essentially the same technique has been used at the level of vertex operators. 


\section{DISCUSSION}

String amplitudes beyond the tree level have been the subject of this paper. Our approach involves conformal field theory, BRST invariance and the use of reparametrization ghosts. Bosonization has been employed to treat spin fields, among others. We have considered all higher-order corrections through one loop, including for example mixed open- and closed-string transitions and contributions from nonorientable surfaces.

Our main purpose has been to present a set of rules so that string theorists can quickly and efficiently calculate corrections to tree-level amplitudes. The presentation has been sufficiently general to cover string theories in their critical dimension and those based on toroidal compactification schemes. The methods are immediately applicable to open or closed strings, whether oriented or unoriented, and whether bosonic, supersymmetric or heterotic.

Several interesting issues remain. One is the conversion of amplitudes from the CartanWeyl basis to a manifestly Lorentz-covariant basis for the $\mathrm{D}=10$ string theories. There are two possible approaches. Presently, the most powerful one at tree level is the reduction method and its modifications as discussed in ref. [82]. Certain operators, such as $\partial X^{\mu}$ or conformal-weight-one currents associated with global symmetries, can be removed from one-loop correlation functions via Ward identities [55]. The applicability of the techniques in ref. [82] to arbitrary conformal-weight-one fields at higher loops needs further investigation. A second method used at tree level in ref. [78] was the classification of amplitudes according to the possible inner products $\left\langle\lambda \cdot \lambda^{\prime}\right\rangle$, where $\lambda$ are the vectors in Eq. (I.1). Different possibilities were called cases. Subsequently, pole and Fierz identities were needed to complete the conversion into manifestly Lorentz-covariant notation. It would be interesting to see if the case analysis is applicable at the one-loop level. Even if this were possible, one would need the analog of pole identities, i.e., certain relations among $\Theta$ functions.

Superstring amplitudes in light-cone gauge for massless particles have been computed 
in ref. [27]. The lattice contribution is similar to Eq. (IV.15) except that the product is from $\mathrm{i}=1$ to 4 instead of $\mathrm{i}=1$ to 6 . The boson associated with bosonization of $\psi^{9}, \psi^{10}$ and the ghost boson $\phi$ are missing. For amplitudes with four or fewer particles, Atick and Sen [59] have discovered a $\theta$-function identity showing that the covariant result agrees with the light-cone result. For more than four vertex operators and for excited states, similar identities should exist. Finding these identities and establishing the equivalence of covariant and light-cone formalisms is an unsolved problem.

Such identities lead to the vanishing of certain amplitudes. For example, for $\mathrm{m} s 0(8)$ fields associated with an internal-symmetry algebra, a Riemann identity [113] leads to

$$
\begin{aligned}
& \sum_{[\alpha, \beta]}(-1)^{2(\alpha+\beta)} \prod_{i=1}^{1} \Theta\left[\begin{array}{l}
\alpha \\
\beta
\end{array}\right]\left(\sum_{\ell} \lambda_{l}^{i} \nu_{\ell}\right)= \\
& 2 \Theta\left[\begin{array}{c}
\frac{1}{2} \\
\frac{1}{2}
\end{array}\right]\left(\frac{1}{2} \sum_{\ell}\left(\lambda_{\ell}^{1}+\lambda_{l}^{2}+\lambda_{\ell}^{3}+\lambda_{\ell}^{4}\right) \nu_{\ell}\right) \quad \Theta\left[\begin{array}{l}
\frac{1}{2} \\
\frac{1}{2}
\end{array}\right]\left(\frac{1}{2} \sum_{\ell}\left(\lambda_{l}^{1}+\lambda_{\ell}^{2}-\lambda_{\ell}^{3}-\lambda_{\ell}^{4}\right) \nu_{\ell}\right)
\end{aligned}
$$

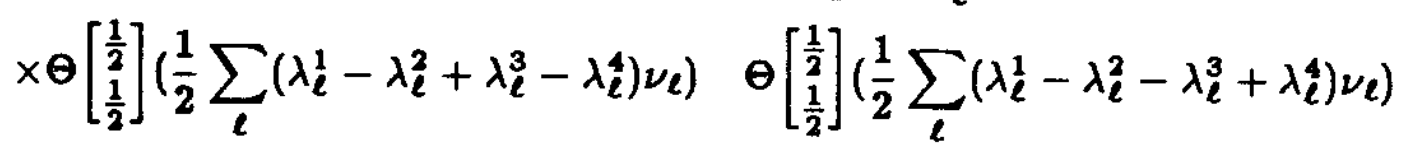

where $\lambda_{\ell}^{i}, \ell=1, \ldots, m, i=1, \ldots, 4$, are the components of the vector $\lambda_{\ell}$ associated with the $\ell^{\text {th }}$ vertex operator. In Eq. (VII.1), $\alpha$ and $\beta$ are 0 or $\frac{1}{2}$. Let us specialize to the GSO-accepted spin fields, i.e., $\lambda_{\ell} \epsilon(s), \lambda_{\ell}^{i}= \pm \frac{1}{2}$, and take $\sum_{\ell} \lambda_{\ell}^{i}=0$. Using $\Theta\left[\frac{\frac{1}{2}}{\frac{1}{2}}\right](0)=0$, an inspection reveals that the right-hand side of Eq. (VII.1) is zero unless each of the eight possible so(8) helicities is present. Thus, $\left\langle S^{\alpha_{m}}\left(z_{m}\right) \ldots . S^{\alpha_{1}}\left(z_{1}\right)\right\rangle=0$ for $m<8$. Moreover, for $m=8$, the Koba-Nielsen-like term cancels the lattice sum to a factor of $\left[\Theta^{\prime}\left[\begin{array}{l}\frac{1}{2} \\ \frac{1}{2}\end{array}\right](0, \tau)\right]^{4} \propto \eta^{12}(\tau)$. This leads to cancellations among $\eta(\tau)$ factors that greatly simplify the result. This phenomenon is well-known from the light-cone formulation where, however, it occurs for completely different reasons [27].

For one-, two-, and three-point amplitudes, whether bosonic, fermionic, or mixed, the lattice sum in Eqs. (IV.16) and (IV.18) reduces to the light-cone case, i.e., N=4. From the above, it then follows that fermionic amplitudes up to three points vanish. With similar 
reasoning, the same can be proved for other amplitudes with up to three legs, again on the basis of the Riemann identity Eq. (VII.1) [40, 58,59]. It would be desirable to have enough Riemann-like theta-function identities to express amplitudes in the most compact form.

Perhaps the most important issue remaining is the extension of the methods presented to higher loops. For the amplitudes in this paper, many different techniques were employed: boundary operators, the method of images, calculation of lattice sums, etc. The simplest derivations occurred with different techniques for different topologies. Can one find effective methods for treating the maze of possibilities occurring in the generic case of a topology with arbitrary numbers of holes, handles and crosscaps? Further investigation along these lines is needed. 


\section{ACKNOWLEDGEMENTS}

We thank Bunji Sakita for discussions concerning mixed open- and closed-string transitions and concerning the normal-ordering prescriptions of vertex operators. We also thank Allan Edmonds and John Ewing for sharing their knowledge of topology. We enjoyed conversations with Wolfgang Lerche, Peter Moxhay, and Andrew Parkes. V.A.K. and S.S. are supported in part by the United States Department of Energy under contracts DE-AC02-84ER40125 and DE-AC02-83ER40107, respectively. 


\section{APPENDIX}

This Appendix discusses the harmonic oscillator. After decomposing $X^{\mu}$ in modes via Eqs. (I.2) and (I.4), the system separates into an infinite number of uncoupled oscillators. The $X^{\mu}$ Green functions can therefore be determined if the harmonic-oscillator case is solved.

The action and hamiltonian are

$$
\begin{aligned}
S_{c l} & =\frac{m}{2} \int_{0}^{T}\left(\dot{X}^{2}+w^{2} X^{2}\right) d \\
H & =\frac{p^{2}}{2 m}+\frac{m w^{2} X^{2}}{2}
\end{aligned}
$$

from which one can compute the propagator [121]

$$
\begin{aligned}
g\left(\tau^{\prime}, \tau\right) & =\left\langle X\left(\tau^{\prime}\right) X(\tau)\right\rangle \\
& =\frac{\int d X_{0} \int_{X(0)=X(T)=X_{0}} D X \exp \left[-S_{c l}(X(\cdot))\right] X\left(\tau^{\prime}\right) X(\tau)}{\int d X_{0} \int_{X(0)=X(T)=X_{0}} D X \exp \left[-S_{c l}(X(\cdot))\right]} \\
& =\frac{\cosh \left[w\left(\left|\tau^{\prime}-\tau\right|-\frac{T}{2}\right)\right]}{2 m w \sinh \left(\frac{w T}{2}\right)}
\end{aligned}
$$

It satisfies the equation

$$
\frac{\partial^{2} g}{\partial \tau^{\prime 2}}=-\frac{1}{m} \delta\left(\tau^{\prime}-\tau\right)+w^{2} g
$$

The contribution to the trace is

$$
\operatorname{Tr}^{-T\left(B-\frac{\phi}{2}\right)}=\left(1-e^{-w T}\right)^{-1}
$$

The propagator for the center-of-mass coordinates of $X^{\mu}$, i.e., for the zero modes, is obtained from (A.1) - (A.3) by taking the limit $\omega \rightarrow 0$. The propagator satisfies the equation

$$
\frac{\partial^{2} g}{\partial \tau^{\prime 2}}=-\frac{1}{m} \delta\left(\tau^{\prime}-\tau\right)+\frac{1}{m T}
$$


The last term in Eq. (A.5) leads to the anholomorphic term in the $X^{\mu}$ propagator:

$$
g\left(\tau^{\prime}, \tau\right)=-\frac{1}{2}\left|\tau^{\prime}-\tau\right|+\frac{\left|\tau^{\prime}-\tau\right|^{2}}{2 m T}
$$

For a fermionic-oscillator system, use anticommuting degrees of freedom. For example,

$$
\operatorname{Tr}_{\text {graded }} e^{-T\left(H+\frac{\phi}{2}\right)}=\operatorname{Tr}(-1)^{F} e^{-T\left(H+\frac{\phi}{2}\right)}=\left(1-e^{-w T}\right)
$$

where $H=\omega\left(d^{\dagger} d-d d^{\dagger}\right)$ and $\left\{d, d^{\dagger}\right\}=1$.

The anholomorphic term in Eq. (IV.4) is due to the zero modes in $X^{\mu}$. First note that

$$
\exp \left(i k x_{0}\right) \exp \left(2 \alpha^{\prime} k p \tau\right)=\exp \left(-\alpha^{\prime} k^{2} \tau\right) \exp [k \cdot x(\tau)]
$$

where $x(\tau)=i x_{0}+2 \alpha^{\prime} p \tau$ is the zero-mode piece of $X_{o p e n}^{\mu}$. A correlation function of exponentials of $X^{\mu}$ contains the factor

$$
\begin{aligned}
\exp \left[-\alpha^{\prime} \sum_{j=1}^{n} k_{j}^{2} \tau_{j}\right] \int \frac{d^{D} k_{0}}{(2 \pi)^{D}}\left\langle k_{0}\left|e^{k_{n} \cdot X\left(r_{n}\right)} \ldots e^{k_{1} \cdot X\left(r_{1}\right)}\right| k_{0}\right\rangle \\
=\exp \left[-\alpha^{\prime} \sum_{j=1}^{n} k_{j}^{2} \tau_{j}\right] \int \frac{d^{D} k_{0}}{(2 \pi)^{D}} \exp \left[-\alpha^{\prime}\left(\tau-\tau_{n}\right)\left(k_{n}+k_{n-1}+\ldots+k_{0}\right)^{2}\right. \\
\left.-\alpha^{\prime} \sum_{j=2}^{n}\left(\tau_{j}-\tau_{j-1}\right)\left(k_{j-1}+\ldots+k_{0}\right)^{2}-\alpha^{\prime} \tau_{1} k_{0}^{2}\right] \\
=\int \frac{d^{D} k_{0}}{(2 \pi)^{D}} \exp \left[-\alpha^{\prime} T k_{0}^{2}+2 \alpha^{\prime} \sum_{j>i} k_{j} \cdot k_{i} \tau_{j}+2 \alpha^{\prime} k_{0} \sum_{j} k_{j} \tau_{j}\right] \\
=\left(\frac{1}{4 \pi \alpha^{\prime} T}\right)^{D / 2} \prod_{j>i}\left\{\left|z_{j}\right|^{2 \alpha^{\prime} k_{j} \cdot k_{i}} \exp \left[-\frac{\alpha^{\prime}}{T} k_{j} \cdot k_{i}\left(\ln \left|z_{j}\right|-\ln \left|z_{i}\right|\right)^{2}\right]\right\}
\end{aligned}
$$

Here, the identity

$$
-\sum_{j>i} k_{j} \cdot k_{i}\left(\tau_{j}-\tau_{i}\right)^{2}=2 \sum_{j>i} k_{j} \cdot k_{i} \tau_{j} \tau_{i}+\sum_{j} k_{j}^{2} \tau_{j}^{2}=\left(\sum_{j} k_{j} \tau_{j}\right)^{2}
$$


has been used, which makes use of $\sum_{j} k_{j}=0$. In Eq. (A.9), $\ln \left|z_{j}\right|=\tau_{j}$. The first equality in (A.9) is obtained by evolving the system with the hamiltonian $H=\alpha^{\prime} p^{2}$. Eq. (A.9) is Eq. (IV.7). Eq. (IV.8) follows for the case when all $k_{j}=0$. 


\section{REFERENCES}

1. M.B. Green and J.H. Schwarz, Phys. Lett. B 149 (1984) 117; Nucl. Phys. B255 (1985) 93.

2. D.J. Gross, J.A. Harvey, E. Martinec and R. Rohm, Phys. Rev. Lett. 54 (1985) 502; Nucl. Phys. B256 (1985) 253; B267 (1986) 75.

3. M.B. Green and J.H. Schwarz, Phys. Lett. B151 (1985) 21.

4. L. Clavelli, Phys. Rev. D33 (1986) 1098.

5. P.H. Frampton, P. Moxhay and Y.J. Ng, Phys. Rev. Lett. 55 (1985) 2107.

6. H. Itoyama and P. Moxhay, Indiana University preprint IUHET 118, to appear in Nucl. Phys. B.

7. D. Amati, C. Bouchiat and J.-L. Gervais, Nuovo Cim. Lett. 2 (1969) 399.

8. K. Bardakci, M.B. Halpern and J.A. Shapiro, Phys. Rev. 185 (1969) 1910.

9. M. Kaku and C.B. Thorn. Phys. Rev. D1 (1970) 2860.

10. G. Frye and L. Susskind, Phys. Lett. 31B (1970) 589.

11. D.J. Gross, A. Neveu, J. Scherk and J.H. Schwasz, Phys. Rev. D2 (1970) 697.

12. A. Neveu and J. Scherk, Phys. Rev. D1 (1970) 2355.

13. G. Frye and L. Susskind, Phys. Lett. 31B (1970) 537.

14. P. Goddard and R.E. Waltz, Nucl. Phys. B34 (1971) 99.

15. V. Alessandrini, D. Amati, M. LeBellac, and D. Olive, Phys. Reports 1C (1971) 269.

16. V. Alessandrini and D. Amati, Nuovo Cim. 4A (1971) 793; V. Alessandrini, D. Amati, and B. Morel, Nuovo Cim. 7A (1972) 797.

17. J.A. Shapiro, Phys. Rev. D5 (1972) 1945.

18. A. Neveu and J. Scherk, Nucl. Phys. B36 (1972) 317.

19. E. Cremmer and J. Scherk, Nucl. Phys. B50 (1972) 222.

20. L. Brink and D.I. Olive, Nucl. Phys. B56 (1973) 253; B58 (1973) 237.

21. L. Clavelli and J.A. Shapiro, Nucl. Phys. B57 (1973) 490. 
22. M.B. Green, Phys. Lett. 46B (1973) 392.

23. L. Brink and D. Fairlie, Nucl. Phys. B74 (1974) 321.

24. J. Winnberg, Nucl. Phys. B94 (1975) 205.

25. M. Ademollo, R. D'Auria, A. D'Adda, F. Gliozzi, E. Napolitano, S. Sciuto, and P. diVecchia, Nucl. Phys. B94 (1975) 221.

26. M. Green and J. Shapiro, Phys. Lett. 64B (1976) 454.

27. J.H. Schwarz, Phys. Rep. 89 (1982) 223.

28. A.M. Polyakov, Phys. Lett. 103B (1981) 207.

29. A.M. Polyakov, Phys. Lett. 103B (1981) 211.

30. O. Alvarez, Nucl. Phys. B216 (1983) 125.

31. D. Friedan, p.839 in J.-B. Zuber and R. Stora, eds., 1982 Les Houches Summer School (North-Holland, Amsterdam, 1984).

32. G. Moore and P. Nelson, Nucl. Phys. B266 (1986) 58.

33. A. Cohen, G. Moore, P. Nelson and J. Polchinski, Nucl. Phys. B267 (1986) 143.

34. J. Polchinski, Commun. Math. Phys. 104 (1986) 37.

35. G. Moore, P. Nelson and J. Polchinski, Phys. Lett. 169B (1986) 47.

36. L. Alvarez-Gaumé, G. Moore and C. Vafa, Commun. Math. Phys. 106 (1986) 1.

37. E. D'Hoker and D.H. Phong, Commun. Math. Phys. 104 (1986) 537.

38. F. Gava, R. Iengo, T. Jayaraman and R. Ramachanchan, "Multiloop Divergences in the Closed Bosonic String Theory" ISAS preprint 90/85/E.P.

39. M. A. Namazie and S. Rajeev, CERN preprint TH.4327/85 (November, 1985).

40. M.A. Namazie, K.S. Narain and M.H. Sarmadi, Phys. Lett. B177 (1986) 329.

41. J.B. Bost and P. Nelson, "Spin-1/2 Bosonization on Compact Surfaces", HUTP86/A044 and Phys. Rev. Lett. 57 (1986) 795.

42. E. D'Hoker and D.H. Phong, Nucl. Phys. B278 (1986) 225; Nucl. Phys. B269 (1986) 205 . 
43. K. Miki, "Fermionic Strings: zero modes and supermoduli" U. of Toky.o preprint, UT-495 (1986).

44. S.R. Das and S.-J. Rey, "Dilation Condensates and Loop Effects in Open and Closed Bosonic Strings" CALT-68-1389 (1986).

45. C.P. Burgess and T.R. Morris, "Open and Unoriented Strings à la Polyakov," IAS preprint (April 1986).

46. C.P. Burgess and T.R. Morris, "Open Superstrings à la Polyakov," IAS preprint (May, 1986).

47. C.P. Burgess, "Finiteness and Type I Superstring" IAS preprint (July, 1986).

48. M.J. Dugan and H. Sonoda, "Functional Determinants on a Riemann Surface," LBL22776 (January, 1987).

49. I. Inami, H. Kanno and T. Kubota, "One Loop Path Integral of the Open Superstring," Osaka U. Preprint OU-HET-98 (Sept., 1986).

50. T. Eguchi and H. Ooguri, "Chiral Bosonization on Riemann Surface," LPTENS preprint (Dec., 1986).

51. E. Verlinde and H. Verlinde, Utrecht preprint (Oct., 1986) "Chiral Bosonization, Determinants and the String Partition Function".

52. E. Verline and H. Verlinde, Utrecht preprint (Jan., 1987) "Multiloop Calculations in Covariant Superstring Theory".

53. B. Grinstein and M.B. Wise, Phys. Rev. D35 (1987) 655; M.R. Douglas and B. Grinstein, Phys. Lett 183B (1987) 52.

54. E. Martinec, Nucl. Phys. 281 (1987) 157.

55. T. Eguchi and H. Ooguri, Nucl. Phys. B282 (1987) 308.

56. D. Gross and P.F. Mende, Princeton U. preprint PUPT-1051 (Feb. 1987).

57. C. Vafa, HUTP-87/A008, "Operator Formulation on Riemann Surfaces".

58. J.J. Atick and A. Sen, Nucl. Phys. B286 (1987) 189.

59. J.J. Atick and A. Sen, SLAC-PUB-4185 (Jan. 1987) "Covariant One Loop Fermion 
Emission Amplitudes in Closed String Theories".

60. J.J. Atick, L.J. Dixon and A. Sen, SLAC-PUB-4235 (Feb., 1987) "String Calculation of Fayet-Iliopoulos D-Terms in Arbitrary Supersymmetric Compactifications".

61. J.J. Atick and A. Sen, SLAC-PUB-4292 (April, 1987) "Two Loop Dilaton Tadpole Induced by Fayet-Illiopoulos D-Terms in Compactifed Heterotic String Theories".

62. W. Lerche, B.E.W. Nilsson and A.W. Schellekens, CERN-TH.4640/87 (Jan., 1987) "Heterotic String-Loop Calculations of the Anomaly Cancelling Term".

63. M. Dine, I. Ichinose and N. Seiberg, IASSNS-HEP-87/87 (Mar., 1987) IAS preprint "F Terms and D Terms in String Theory".

64. J.-L. Gervais and B. Sakita, Nucl. Phys. B34 (1971) 477.

65. L. Brink, D.I. Olive and J. Scherk, Nucl. Phys. B61 (1973) 173.

66. A.A. Belavin, A.M. Polyakov and A.B. Zamolodchikov, Nucl. Phys. B241 (1984) 333.

67. D. Friedan, Z. Qiu and S. Shenker, p. 419 in J. Lepowsky, S. Mandelstam and I.M. Singer, eds., "Vertex Operators in Mathematics and Physics" (Springer-Verlag, 1984).

68. V.G. Knizhnik and A.B. Zamolodchikov, Nucl. Phys. B247 (1984) 83.

69. M.A. Bershadsky, V.G. Knizhnik and M.G. Teitelman, Phys. Lett. 151B (1985) 31.

70. D. Friedan, Z. Qiu and S. Shenker, Phys. Lett. 151B (1985) 37.

71. D. Friedan, S. Shenker and E. Martinec, Phys. Lett. 160B (1985) 55.

72. V.G. Knizhnik, Phys. Lett. 160B (1985) 403.

73. D. Friedan, E. Martinec and S. Shenker, Nucl. Phys. B271 (1986) 93.

74. J. Cohn, D. Friedan, Z. Qiu and S. Shenker, Nucl. Phys. B278 (1986) 577.

75. V.G. Knizhnik, L.D. Landau Institute preprint (June, 1986).

76. V.A. Kostelecký, O. Lechtenfeld, W. Lerche, S. Samuel and S. Watamura, Phys. Lett. B 182 (1986) 311.

77. V.A. Kostelecký, O. Lechtenfeld, S. Samuel, D. Verstegen, S. Watamura, and D. Sahdev, Phys. Lett. B 183 (1987) 299. 
78. V.A Kostelecký, O. Lechtenfeld, W. Lerche, S. Samuel and S. Watamura, Nucl. Phys. B288 (1987) 173.

79. M. Kato and K. Ogawa, Nucl. Phys. B212 (1983) 443.

80. S. Hwang, Phys. Rev. D 28 (1983) 2614.

81. V.A. Kostelecký and O. Lechtenfeld, Phys. Rev. Lett. 59 (1987) 169.

82. S. Samuel, "An Infinite Set of Conservation Laws for the Ghost-Extended Superstring," Nucl. Phys. B, in press.

83. M.B. Halpern, Phys. Rev. D12 (1975) 1684.

84. T. Banks, D. Horn and M. Neuberger, Nucl. Phys. B108 (1976) 119.

85. A. Feingold and J. Lepowsky, Adv. Math. 29 (1978) 271.

86. J. Lepowsky and R.L. Wilson, Commun. Math. Phys. 62 (1978) 43.

87. I. Frenkel and V.G. Kac, Invent. Math. 62 (1980) 23.

88. G. Segal, Commun. Math. Phys. 80 (1981) 301.

89. P. Goddard and D.I. Olive, p. 51 in J. Lepowsky, S. Mandelstam and I.M. Singer, eds., "Vertex Operators in Mathematics and Physics" (Springer-Verlag, 1984).

90. I.B. Frenkel, J. Lepowsky and A. Meurman, p. 231 in J. Lepowsky, S. Mandelstam and I.M. Singer, eds., "Vertex Operators in Mathematics and Physics" (Springer-Verlag, 1984).

91. I.B. Frenkel, J. Lepowsky and A. Meurman, Contemp. Math. 45 (1985) 99.

92. L. Alvarez-Gaumé, J.-B. Bost, G. Moore, P. Nelson and C. Vafa, Phys. Lett. B178 (1986) 41.

93. L. Alvarez-Gaumé, J.-B. Bost, G. Moore, P. Nelson and C. Vafa, "Bosonization on Higher Genus Riemann Surfaces”, HUTP-86/A062 CERN-TH 4660/87 (March, 1987).

94. L. Alvarez-Gaumé, C. Gomez and C. Reina, "Loop groups, grassmanians and string theory" CERN-TH.4641/87.

95. W.S. Massey, Algebraic Topology: an Introduction (Harcourt, Brace and World, New York, 1967). 
96. M. Schiffer and D.C. Spencer, Functionals of Finite Riemann Surfaces (Princeton University Press, Princeton, 1954).

97. S. Weinberg, Phys. Lett. B 156 (1985) 309.

98. B. Grinstein and R. Rohm, "Dirac and Majorana Spinors on Nonorientable Riemann Surfaces," preprint CALT-68-1421 (1987).

99. C.G. Callan, C. Lovelace, C.R. Nappi and S.A. Yost, "Adding Holes and Crosscaps to the Superstring” PUPT-1045 (March, 1987).

100. J. Polchinski and Y. Cai, "Consistency of Open Superstring Theories," UTTG-09-87 (March, 1987).

101. J.A. Shapiro and C.B. Thorn, "BRST Invariant Transitions Between Closed and Open Strings" IASSNS-HEP87/7 (IAS preprint, 1987).

102. Z. Hlousek and A. Jevicki, Nucl. Phys. B288 (1987) 131.

103. N. Seiberg and E. Witten, Nucl. Phys. B276 (1986) 272.

104. C. Lovelace, Nucl. Phys. B273 (1986) 413.

105. W. Fischler and L. Susskind, Phys. Lett. 173 B (1986) 262.

106. C.G. Callan, C. Lovelace, C. Nappi, and S. Yost, Princeton preprint PUPT-1027 (1986), to appear in Nucl. Phys. B.

107. S. Samuel, "Mathematical Formulation of E. Witten's Superstring Field Theory", Nucl. Phys. B, in press.

108. E. Witten, Nucl. Phys. B268 (1986) 253.

109. E. Witten, Nucl. Phys. B276 (1986) 291.

110. D. Gross and A. Jevicki, Nucl. Phys. B283 (1987) 1.

111. C. Thorn, "Perturbation Theory for Quantized String Fields," IAS preprint; Proceedings of the XXIII International Conference on High-Energy Physics, Berkeley, 1986.

112. D. Gross and A. Jevicki, "Operator Formulation of Interacting String Field Theory II," Santa Barbara preprint NSF-ITP-86-119 (September, 1986).

113. D. Mumford, Tata Lectures on Theta, Vol. I (Birkhauser, Boston, 1983). 
114. P.G.O. Freund, Phys. Lett. 151B (1985) 387.

115. L. Alvarez-Gaumé, P. Ginsparg, G. Moore and C. Vafa, Phys. Lett. B171 (1986) 155.

116. L.J. Dixon and J.A. Harvey, Nucl. Phys. B274 (1986) 93.

117. J. A. Harvey, p. 704 in M.B. Green and D.J. Gross, eds., "Unified String Theories" (World Scientific, Singapore, 1985).

118. H. Kawai, D.C. Lewellen and S.-H. H. Tye, Cornell preprint CLNS 86/732 (May, 1986).

119. W. Lerche, D. Lüst and A. Schellekens, "10-Dimensional Heterotic Strings from Niemeier Lattices," CERN-TH.4540/86; "Chiral 4-Dimensional Heterotic Strings from SelfDual Lattices," CERN-TH.4590/86.

120. S. Samuel, Phys. Lett. B 181 (1986) 255.

121. R.P. Feynman and A.R. Hibbs, Quantum Mechanics and Path Integrals (McGraw-Hill, New York, 1965). 
FIGURE CAPTIONS

\section{Figure 1.}

(a) The annulus of length $T$ is obtained by joining left and right ends, so that $A$ is identified with $B, C$ with $F$, and D with $\mathrm{E}$.

(b) The same in terms of $z=\exp (\rho)$. The points A-F are the images of those in (a). 


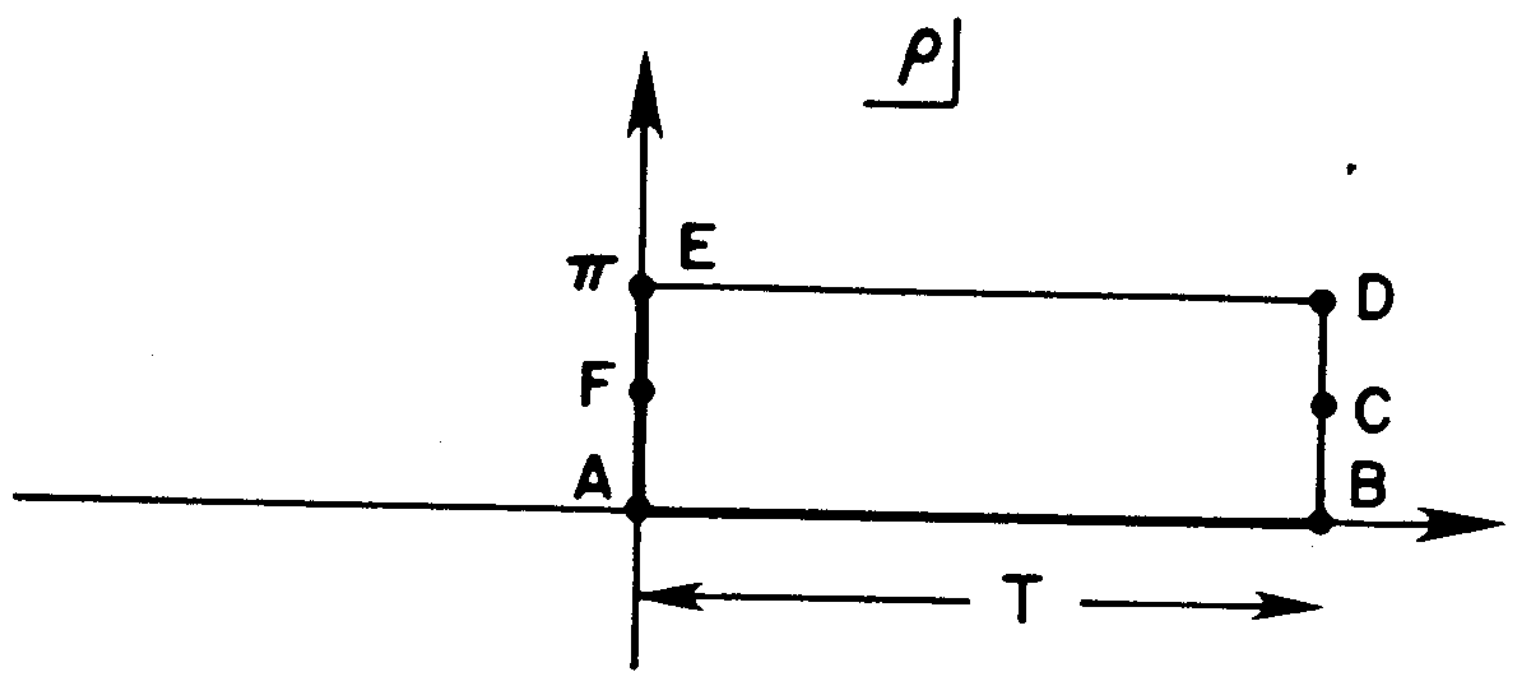

Figure 19

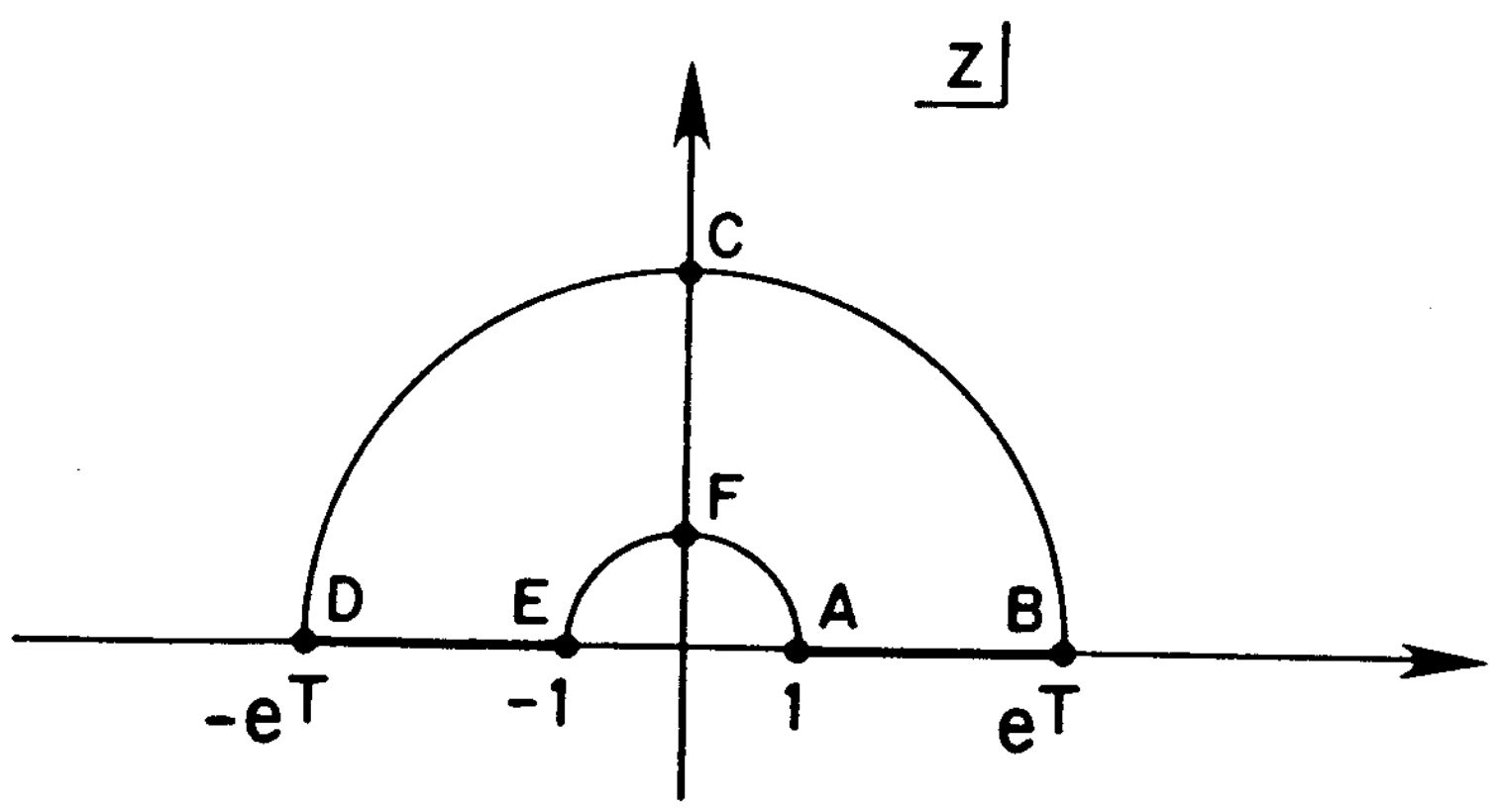

Figure $1 \mathrm{~b}$ 